\title{
48. THE EVOLUTION OF ANTARCTIC SURFACE WATERS DURING THE PALEOGENE: INFERENCES FROM THE STABLE ISOTOPIC COMPOSITION OF PLANKTONIC FORAMINIFERS, ODP LEG $\mathbf{1 1 3}^{1}$
}

\author{
Lowell D. Stott, ${ }^{2,3}$ James P. Kennett, ${ }^{2}$ Nicholas J. Shackleton, ${ }^{4}$ and Richard M. Corfield ${ }^{5}$
}

\begin{abstract}
The oxygen and carbon isotopic composition has been measured for numerous Paleogene planktonic foraminifer species from Maud Rise, Weddell Sea (ODP Sites 689 and 690), the first such results from the Antarctic. The results provide information about large-scale changes in the evolution of temperatures, seasonality, and structure of the upper water column prior to the development of a significant Antarctic cryosphere. The early Paleocene was marked by cooler surface-water conditions compared to the Cretaceous and possibly a less well developed thermocline. The late Paleocene and early Eocene saw the expansion of the thermocline as Antarctic surface waters became warm-temperate to subtropi$\mathrm{cal}$. The late Paleocene to early Eocene thermal maximum was punctuated by two brief excursions during which time the entire Antarctic water column warmed and the meridional temperature gradient was reduced. The first of these excursions occurred at the Paleocene/Eocene boundary, in association with a major extinction in deep sea benthic foraminifers. The second excursion occurred within the early Eocene at $\sim 54.0 \mathrm{Ma}$. These excursions are of global importance and represent the warmest intervals of the entire Cenozoic. The excursions were associated with fundamental changes in deep-water circulation and global heat transport.

The thermal maximum of the early Eocene ended with the initiation of a long-term cooling trend at $52.0 \mathrm{Ma}$. This cooling trend was associated with reduced seasonality, and diminished structure and/or duration of the seasonal thermocline. The cooling trend was punctuated by three major cooling steps at $43.0,40.0$, and $\sim 36.0 \mathrm{Ma}$.
\end{abstract}

\section{INTRODUCTION}

Ocean drilling on Maud Rise, $65^{\circ} \mathrm{S}$ in the Weddell Sea, Antarctica (ODP Sites 689 and 690 ), has provided the first nearly continuous calcareous biogenic records of Paleogene and Upper Cretaceous age from the Antarctic Ocean and, at $65^{\circ} \mathrm{S}$, the highest latitude calcareous sequences yet drilled. Planktonic foraminifers and calcareous nannofossils are abundant throughout the Paleogene and Upper Cretaceous of both Maud Rise sites. Until now insufficient material was available to examine the evolution of surface waters in the Antarctic Ocean in relation to the refrigeration and developing cryosphere within the Antarctic region during the Cenozoic. The only previous material available was from subantarctic latitudes, well north of the water immediately adjacent to the Antarctic continent (Shackleton and Kennett, 1975; Kennett and Shackleton, 1976). The climatic evolution of the Southern Ocean was in part brought about by rearrangement of Southern Hemisphere land masses, and is related to circulation changes that affected global heat transport.

In this paper we report the isotopic changes in Paleogene planktonic foraminifer species of the Weddell Sea and the inferred changes that occurred in the temperature and structure of the upper part of the water column. The major Cenozoic isotopic trends reflecting global changes in the isotopic composition of the oceans have been discussed elsewhere. The major emphasis of this work is to document the environmental changes

\footnotetext{
${ }^{1}$ Barker, P. F., Kennett, J. P., et al., 1990. Proc. ODP, Sci. Results, 113: College Station, TX (Ocean Drilling Program).

2 Marine Science Institute and Department of Geological Sciences, University California, Santa Barbara, CA 93106.

${ }^{3}$ Present address: Department of Geological Sciences, University of Southern California, Los Angeles, CA 90089.

4 University of Cambridge, Godwin Laboratory for Quaternary Research, Free Lane, Cambridge CB2 3RS, United Kingdom.

5 Department of Earth Sciences, University of Oxford, Parks Road, Oxford, OX1 3PR, United Kingdom.
}

that occurred within the Antarctic Ocean. The oxygen isotopic composition of well preserved foraminifers provides a proxy for temperature, while carbon isotopic composition reflects primarily the $\delta^{13} \mathrm{C}$ of dissolved inorganic carbon (DIC) within the water column and the extent to which ${ }^{12} \mathrm{C}$ is depleted through organic productivity. Inferences about water column chemistry and temperature in the Paleogene assumes that the planktonic foraminifers chosen for isotopic analysis secreted their tests close to isotopic equilibrium with their environment. Many modern planktonic foraminifers appear to secrete their tests close to oxygen isotopic equilibrium and therefore, provide a good measure of water column temperature during calcification (see Mix, 1987). However, $\delta^{13} \mathrm{C}$ disequilibrium is usually observed, the extent of which depends on a number of poorly understood factors including the presence of photosymbionts and possibly on the isotopic composition of different food sources (Shackleton et al., 1973; Williams et al., 1977; Spero and DeNiro, 1987; Spero and Williams, 1988). Despite the fact that the measurements themselves do not precisely record water column isotopic values, the relative changes that occurred within a taxonomic group over time probably reflect environmental changes. A number of previous studies have illustrated the relationship between $\delta^{18} \mathrm{O}$ and $\delta^{13} \mathrm{C}$ in planktonic foraminifers (Douglas and Savin, 1973, 1975, 1978; Boersma and Shackleton, 1979; Shackleton et al., 1985; Keigwin and Corliss, 1986). Shallow-dwelling forms are inferred to be those that display the lowest $\delta^{18} \mathrm{O}$ values and the highest $\delta^{13} \mathrm{C}$ values reflecting warmer, nutrient-depleted conditions near the surface. However, because planktonic foraminifers may migrate within the water column (Bé, 1982) their isotopic composition can vary accordingly. Shackleton et al. (1985) showed that Paleocene planktonic foraminifers, particularly those with globorotalid-like morphotypes, exhibit considerable ontogenetic variability in isotopic composition. This was interpreted to reflect migratory patterns during ontogeny. The isotopic/ontogenetic relationships appear to be species-specific. Such studies have generally assumed that planktonic foraminifers in fossil assemblages lived during the same time of the year and that isotopic 
variations between species reflect vertical water column characteristics rather than seasonal patterns in species succession. Sediment trap and plankton tow studies have shown however, that planktonic foraminifers, particularly in regions marked by high seasonality, do not occur continuously nor do all species occur simultaneously during the year. Different species seem to prefer specific nutrient and temperature conditions that can vary seasonally (Bé, 1960; Thunell and Honjo, 1987; Reynolds and Thunell, 1985). Therefore, the occurrence of species and their isotopic composition within the Paleogene fossil assemblages contain a range of seasonal signals that are difficult to decipher because the genera and species have no modern representatives.

In the Antarctic Ocean, because of long winter darkness, the seasonal succession of planktonic foraminifer species during the Paleogene was probably shorter compared with middle and low latitude regions. However, this assumption may be in error if high latitude species were adapted to deeper habitats below the euphotic zone, as some species are today (Smith and Gowing, 1988), and were not necessarily connected with seasonal light/ dark cycles. Furthermore, seasonal sea surface temperature contrasts may have been much higher in the Antarctic because of the long winter darkness. Inferences about water column stratification based on the isotopic differences between species living at slightly different times of the year would result in erroneous interpretations about water column characteristics. In this paper we attempt to address these issues in order to decipher patterns of seasonality change in the Antarctic fossil record. Analyses of different size fractions (ontogenetic stages) of various planktonic species have been carried out to identify migratory behavior that would have influenced the isotopic composition of planktonic foraminifers secreting calcite at different depths within the water column. We have identified the size fraction for each species that provides the lowest $\delta^{18} \mathrm{O}$ and highest $\delta^{13} \mathrm{C}$ values. These various size fractions have been utilized in developing an isotope time series to examine changes in surface-water conditions during the Paleogene. Isotopic differences between the fossil planktonic foraminifer groups are interpreted in terms of vertical stratification of the water column and potential seasonality contrasts. In addition to the longer-term patterns of change, we have also examined specific "events" in the oxygen and carbon isotope stratigraphies which may represent important paleoceanographic and paleoclimatic changes.

\section{METHODS}

Samples of $10-20 \mathrm{~cm}^{3}$ were taken at $\sim 75 \mathrm{~cm}$ intervals from sequences at ODP Sites 689 and 690 . Stratigraphic resolution varies in accordance with changes in sediment accumulation rates. However, sufficient resolution is provided by this sample strategy to define the major patterns of change in the isotopic composition of planktonic foraminifers through the Paleogene.

Each sample was dried overnight in an oven at $50^{\circ} \mathrm{C}$ and weighed. The weighed samples were soaked in either a Calgon solution or a sodium hexametaphosphate solution $(\mathrm{pH}=7)$ for $24 \mathrm{hr}$. Most samples disaggregated readily and could be washed over a $63 \mu \mathrm{m}$ screen. Some samples required longer soaking times before being washed. The $>63 \mu \mathrm{m}$ residue was dried at $50^{\circ} \mathrm{C}$ in an oven and then reweighed. Samples were sieved to obtain four different size fractions: $355-300 \mu \mathrm{m}$ (= size C), 300$250 \mu \mathrm{m}$ (= size D), 250-212 $\mu \mathrm{m}$ (= size E), and 212-180 $\mu \mathrm{m}$ (= size F). From these fractions, species were identified and picked for isotopic analysis. Sample sizes varied between size fractions but generally consisted of between 20 and 40 specimens. The samples were sonicated in methanol for approximately $10 \mathrm{~s}$ to remove adhering microfossils, and then dried at $50^{\circ} \mathrm{C}$. Planktonic foraminifers were transferred to reaction vessels and roasted under vacuum for $1 \mathrm{hr}$ at $375^{\circ} \mathrm{C}$.
Isotopic analyses were carried out at the Graduate School of Oceanography, University of Rhode Island; Lamont-Doherty Geological Observatory; Department of Geological Sciences, University of California, Santa Barbara; and at the Godwin Laboratory, Cambridge University. Interlaboratory calibration is conducted through the analysis of international standards such as NBS 20, NBS 18, and NBS 19. Results are reported relative to the PDB standard for both oxygen and carbon. Precision is $0.1 \%$ or better for both $\delta^{18} \mathrm{O}$ and $\delta^{13} \mathrm{C}$.

The preservation of all the samples analyzed was evaluated under the microscope. Samples discussed here are all considered to be well preserved. No visible sign of recrystallization was observed in the samples analyzed isotopically.

The age model applied to the data set discussed here and used to illustrate the temporal patterns is based upon the planktonic foraminifer biostratigraphy of Stott and Kennett (this volume, chapter 34), which has been correlated to the magnetostratigraphy of Sites 689 and 690 . Ages were determined by interpolating between datum levels, assuming continuous and uniform sedimentation rates. Datum levels used in the development of the age model are given in Table 1 . The temperature equation relating oxygen isotopic composition to the temperature of calcification is based on the equation of O'Neil et al. (1969) and the considerations given in Shackleton (1974). Temperature calculations assume no significant ice accumulation before the early Oligocene. Additionally, it is likely that surface-water salinity varied at high southern latitudes during the Paleogene. This variability complicates any accommodation for salinity effect in the paleotemperature equation and precludes the application of any single correction factor. Therefore, salinity changes could account for errors in our paleotemperature estimates of up to several degrees centigrade. Nevertheless, the record of interspecific isotopic differences and the longer-term isotopic changes should reflect primarily the pattern of paleotemperature history during the Paleogene.

\section{RESULTS}

Results of planktonic foraminifer isotopic analyses from Sites 689 and 690 are provided in Tables 2 and 3. Some samples have been analyzed at several size ranges and the isotopic results for each are presented. Based on the results of these size-specific analyses, specific size fractions of the planktonic foraminifers Acarinina and Subbotina were chosen that represent the lowest $\delta^{18} \mathrm{O}$ and highest $\delta^{13} \mathrm{C}$ values. These data are included with those samples whose multiple size fractions were not analyzed. The data are presented for each site, discussing oxygen and carbon isotopic stratigraphy separately.

\section{Oxygen Isotopes}

\section{Site 690}

Figure 1 shows the oxygen isotopic composition for a number of species of Acarinina and Subbotina. The total temperature range recorded for taxa within the middle Paleocene and middle Eocene is about $10^{\circ} \mathrm{C}$. The species of Acarinina exhibit the lowest $\delta^{18} \mathrm{O}$ values throughout the interval. The lowest values exhibited by this group are approximately $-1.5 \%$ in the early Eocene, while the highest values are $\sim 0.0 \%$ during the middle Paleocene and middle Eocene. Subbotina species consistently exhibit the highest $\delta^{18} \mathrm{O}$ values throughout the sequence. The minimum difference between these two groups is $\sim 0.7 \%$ $\left(\sim 3^{\circ} \mathrm{C}\right)$ in the early Eocene and $\sim 0.3 \% 0\left(\sim 1.5^{\circ} \mathrm{C}\right)$ in the middle to late Paleocene. Isotopically inferred temperatures were never less than $\sim 9^{\circ} \mathrm{C}$ in any of the species analyzed before the middle Eocene; not $<8^{\circ} \mathrm{C}$ in the middle Eocene and $<5^{\circ} \mathrm{C}$ in the late Eocene. 
Table 1. Datums used to develop age models for Sites 689 and 690.

\begin{tabular}{|c|c|c|c|}
\hline Hole & Datum level & $\begin{array}{l}\text { Depth } \\
\text { (mbsf) }\end{array}$ & $\begin{array}{l}\text { Age } \\
\text { (Ma) }\end{array}$ \\
\hline \multirow[t]{19}{*}{ 689B } & Base Chron $8 \mathrm{~N}-2$ & 75.97 & 27.74 \\
\hline & Top Chron 9N & 79.46 & 28.15 \\
\hline & Base Chron $10 \mathrm{~N}$ & 91.93 & 30.33 \\
\hline & Base Chron $11 \mathrm{~N}$ & 103.37 & 32.06 \\
\hline & Base Chron $12 \mathrm{~N}$ & 106.87 & 32.90 \\
\hline & Top Chron $13 \mathrm{~N}$ & 119.69 & 35.87 \\
\hline & Top Chron $16 \mathrm{~N}$ & 128.32 & 38.10 \\
\hline & Base Chron $16 \mathrm{~N}$ & 134.02 & 39.24 \\
\hline & Top Chron $17 \mathrm{~N}-1$ & 135.75 & 39.52 \\
\hline & Base Chron $17 \mathrm{~N}-3$ & 152.73 & 41.11 \\
\hline & Top Chron $18 \mathrm{~N}$ & 153.70 & 41.21 \\
\hline & Base Chron $19 \mathrm{~N}$ & 163.16 & 44.06 \\
\hline & Top Chron $20 \mathrm{~N}$ & 165.55 & 44.66 \\
\hline & Unconformity & 202.25 & \\
\hline & P. australiformis FAD & 207.92 & 57.60 \\
\hline & A. praepentacamerata FAD & 215.30 & 60.25 \\
\hline & Unconformity & 227.51 & \\
\hline & S. inconstans FAD & 229.84 & 64.70 \\
\hline & S. pseudobulloides FCOD & 231.89 & 66.10 \\
\hline \multirow[t]{25}{*}{ 690B } & Top Chron $8 \mathrm{~N}$ & 53.25 & 26.86 \\
\hline & Base Chron $8 \mathrm{~N}$ & 60.12 & 27.74 \\
\hline & Top Chron 9N & 61.00 & 28.15 \\
\hline & Base Chron 9N & 68.47 & 29.21 \\
\hline & Top Chron $10 \mathrm{~N}$ & 72.17 & 29.73 \\
\hline & Base Chron $10 \mathrm{~N}$ & 73.92 & 30.33 \\
\hline & Top Chron $11 \mathrm{~N}$ & 80.75 & 31.23 \\
\hline & Base Chron $11 \mathrm{~N}$ & 84.00 & 32.06 \\
\hline & Top Chron $13 \mathrm{~N}$ & 91.70 & 35.39 \\
\hline & Unconformity & 93.2 & \\
\hline & Base Chron $16 \mathrm{~N}$ & 95.70 & 39.24 \\
\hline & Top Chron $17 \mathrm{~N}$ & 96.74 & 39.53 \\
\hline & Base Chron $18 \mathrm{~N}-1$ & 105.67 & 41.73 \\
\hline & Unconformity & 106.77 & \\
\hline & $\sim$ Top Chron $20 \mathrm{~N}$ & 107.36 & 44.69 \\
\hline & Unconformity & 118.36 & \\
\hline & $\sim$ Top Chron $2 \mathrm{iN}$ & 118.36 & 49.11 \\
\hline & Base Chron $21 \mathrm{~N}$ & 123.63 & 50.34 \\
\hline & Base Chron $22 \mathrm{~N}$ & 133.17 & 52.62 \\
\hline & Top Chron $23 \mathrm{~N}$ & 137.20 & 53.88 \\
\hline & Base Chron $23 \mathrm{~N}$ & 140.87 & 54.70 \\
\hline & Top Chron $24 \mathrm{~N}$ & 144.42 & 55.14 \\
\hline & Base Chron 24N & 154.62 & 56.14 \\
\hline & Top Chron $25 \mathrm{~N}$ & 185.47 & 58.64 \\
\hline & Top Chron $26 \mathrm{~N}$ & 210.05 & 60.21 \\
\hline
\end{tabular}

\section{Acarininid Group}

Clear temporal trends are evident in the oxygen isotope stratigraphy of both taxonomic groups (acarininids and subbotinids). These trends reflect major changes in the surface-water temperature profile within the Antarctic Ocean during the early Paleogene. Maximum surface-water temperatures, as expressed in the Acarinina isotope profile, were $\sim 12^{\circ} \mathrm{C}$ during the middle $\mathrm{Pa}$ leocene and show a trend toward warmer temperatures of about $14^{\circ} \mathrm{C}$ by the latest Paleocene, $(\sim 58.0 \mathrm{Ma})$. Near the boundary between the Paleocene and Eocene the planktonic oxygen isotopic temperatures exhibit a pronounced excursion superimposed upon the longer-term warming trend that began in the middle Paleocene (Fig. 1). This excursion is remarkable because it occurred rapidly, within 200 k.y. and represents one of the warmest peaks $\left(>20^{\circ} \mathrm{C}\right)$ in Antarctic temperatures during the entire Late Cretaceous and Cenozoic. The spike is recorded throughout the water column (Kennett and Stott, this volume). The excursion also represents the beginning of a period of general surface-water warmth that spanned an interval of 8.0 m.y. in the early Eocene. However, there is a small reduction in surface-water temperatures following the maximum at the Paleocene/Eocene boundary (Fig. 1). Within this warm interval, during the early Eocene, another maximum occurs at $\sim 54.0 \mathrm{Ma}$ at which
Table 2. Planktonic foraminifer isotope data for Hole 689B.

\begin{tabular}{|c|c|c|c|c|}
\hline $\begin{array}{c}\text { Species } \\
\text { (size fraction) }\end{array}$ & $\begin{array}{l}\text { Depth } \\
\text { (mbsf) }\end{array}$ & $\begin{array}{l}\text { Age } \\
\text { (Ma) }\end{array}$ & $\delta^{18} \mathrm{O}$ & $\delta^{13} \mathrm{C}$ \\
\hline \multirow[t]{61}{*}{ Subbotina angiporoides ( $\mathrm{E}$ and $\mathrm{F}$ ) } & 102.10 & 31.86 & 2.16 & 0.26 \\
\hline & 102.85 & 31.98 & 2.27 & 0.55 \\
\hline & 102.85 & 31.98 & 2.16 & 0.83 \\
\hline & 104.35 & 32.28 & 2.05 & 1.35 \\
\hline & 104.35 & 32.28 & 2.30 & 1.10 \\
\hline & 105.85 & 32.63 & 2.22 & 1.53 \\
\hline & 105.85 & 32.63 & 2.53 & 1.38 \\
\hline & 105.85 & 32.63 & 2.49 & 1.26 \\
\hline & 106.60 & 32.81 & 2.36 & 1.49 \\
\hline & 106.60 & 32.81 & 2.59 & 1.28 \\
\hline & 106.60 & 32.81 & 2.43 & 1.28 \\
\hline & 107.35 & 32.99 & 2.08 & 1.08 \\
\hline & 108.10 & 33.17 & 2.31 & 1.40 \\
\hline & 108.10 & 33.17 & 2.62 & 1.16 \\
\hline & 108.10 & 33.17 & 2.60 & 1.23 \\
\hline & 108.85 & 33.35 & 2.06 & 0.89 \\
\hline & 109.60 & 33.53 & 1.94 & 0.78 \\
\hline & 110.95 & 33.86 & 2.40 & 0.96 \\
\hline & 112.44 & 34.23 & 2.18 & 1.45 \\
\hline & 112.44 & 34.23 & 2.54 & 1.18 \\
\hline & 112.44 & 34.23 & 2.22 & 1.42 \\
\hline & 113.21 & 34.41 & 2.28 & 1.10 \\
\hline & 113.95 & 34.60 & 2.53 & 1.31 \\
\hline & 114.71 & 34.78 & 2.47 & 1.22 \\
\hline & 115.43 & 34.96 & 2.45 & 1.14 \\
\hline & 116.20 & 35.14 & 2.59 & 1.32 \\
\hline & 116.94 & 35.32 & 2.37 & 1.85 \\
\hline & 116.94 & 35.32 & 2.67 & 1.71 \\
\hline & 117.71 & 35.47 & 2.67 & 1.48 \\
\hline & 118.45 & 35.61 & 2.68 & 1.90 \\
\hline & 119.22 & 35.76 & 2.56 & 1.56 \\
\hline & 120.57 & 36.07 & 1.94 & 1.55 \\
\hline & 121.30 & 36.26 & 0.28 & 0.50 \\
\hline & 122.06 & 36.46 & 1.68 & 1.64 \\
\hline & 122.80 & 36.65 & 0.52 & 0.61 \\
\hline & 123.56 & 36.84 & 1.64 & 1.41 \\
\hline & 124.30 & 37.04 & 1.71 & 1.37 \\
\hline & 125.06 & 37.23 & 1.73 & 1.69 \\
\hline & 125.82 & 37.43 & 1.84 & 1.47 \\
\hline & 128.08 & 38.02 & 1.30 & 1.48 \\
\hline & 128.84 & 38.19 & 1.40 & 1.20 \\
\hline & 131.65 & 38.76 & 1.53 & 1.38 \\
\hline & 132.37 & 38.91 & 1.30 & 1.46 \\
\hline & 133.15 & 39.07 & 1.58 & 1.50 \\
\hline & 133.87 & 39.21 & 1.41 & 1.37 \\
\hline & 134.65 & 39.33 & 1.57 & 1.33 \\
\hline & 135.37 & 39.44 & 0.39 & 0.30 \\
\hline & 136.15 & 39.54 & 1.47 & 1.09 \\
\hline & 137.65 & 39.69 & 1.63 & 1.23 \\
\hline & 138.37 & 39.75 & 1.56 & 0.86 \\
\hline & 138.37 & 39.75 & 1.57 & 1.26 \\
\hline & 140.50 & 39.95 & 1.43 & 0.99 \\
\hline & 141.25 & 40.02 & 1.48 & 1.12 \\
\hline & 142.75 & 40.16 & 1.61 & 1.18 \\
\hline & 143.50 & 40.24 & 1.63 & 1.16 \\
\hline & 144.25 & 40.31 & 1.53 & 1.05 \\
\hline & 145.00 & 40.38 & 1.44 & 0.98 \\
\hline & 145.75 & 40.45 & 1.53 & 0.97 \\
\hline & 155.45 & 41.72 & 1.07 & 1.23 \\
\hline & 157.70 & 42.42 & 1.10 & 1.31 \\
\hline & 158.45 & 42.65 & 0.80 & 1.11 \\
\hline \multirow[t]{17}{*}{ Subbotina eocaenica (E and F) } & 126.58 & 37.63 & 1.97 & 1.49 \\
\hline & 139.15 & 39.83 & 1.94 & 1.07 \\
\hline & 142.00 & 40.09 & 1.47 & 1.31 \\
\hline & 144.25 & 40.31 & 1.70 & 1.20 \\
\hline & 146.50 & 40.52 & 1.64 & 1.24 \\
\hline & 147.58 & 40.62 & 0.82 & 1.01 \\
\hline & 148.00 & 40.66 & 0.83 & 0.94 \\
\hline & 148.75 & 40.73 & 1.21 & 1.19 \\
\hline & 149.45 & 40.80 & 1.39 & 1.29 \\
\hline & 149.97 & 40.85 & 1.20 & 1.28 \\
\hline & 150.95 & 40.94 & 1.27 & 1.33 \\
\hline & 151.47 & 40.99 & 1.30 & 1.26 \\
\hline & 151.70 & 41.01 & 1.22 & 1.16 \\
\hline & 152.45 & 41.08 & 1.41 & 1.26 \\
\hline & 152.97 & 41.13 & 1.39 & 1.29 \\
\hline & 153.20 & 41.15 & 1.24 & 1.12 \\
\hline & 153.95 & 41.26 & 1.28 & 1.24 \\
\hline
\end{tabular}


Table 2 (continued).

\begin{tabular}{|c|c|c|c|c|}
\hline $\begin{array}{c}\text { Species } \\
\text { (size fraction) }\end{array}$ & $\begin{array}{l}\text { Depth } \\
\text { (mbsf) }\end{array}$ & $\begin{array}{l}\text { Age } \\
\text { (Ma) }\end{array}$ & $\delta^{18} \mathrm{O}$ & $\delta^{13} \mathrm{C}$ \\
\hline \multirow[t]{34}{*}{ Subbotina eocaenica (E and F) } & 154.47 & 41.42 & 1.11 & 1.11 \\
\hline & 154.70 & 41.49 & 1.17 & 1.24 \\
\hline & 155.97 & 41.88 & 1.37 & 1.38 \\
\hline & 156.20 & 41.95 & 1.29 & 1.28 \\
\hline & 156.95 & 42.19 & 1.31 & 1.27 \\
\hline & 158.45 & 42.65 & 1.12 & 1.57 \\
\hline & 161.40 & 43.56 & 1.04 & 1.58 \\
\hline & 162.16 & 43.80 & 0.71 & 1.40 \\
\hline & 162.90 & 44.03 & 0.60 & 1.19 \\
\hline & 178.45 & 46.27 & 0.46 & 0.74 \\
\hline & 179.19 & 46.36 & 0.79 & 0.96 \\
\hline & 179.95 & 46.46 & 0.50 & 0.68 \\
\hline & 180.96 & 46.55 & 0.79 & 0.92 \\
\hline & 182.95 & 46.83 & 0.39 & 1.17 \\
\hline & 189.65 & 47.65 & 0.27 & 0.83 \\
\hline & 190.40 & 47.74 & 0.16 & 0.55 \\
\hline & 191.15 & 47.82 & 0.45 & 0.96 \\
\hline & 191.90 & 47.91 & 0.40 & 1.06 \\
\hline & 197.85 & 48.59 & 0.24 & 1.26 \\
\hline & 198.60 & 48.68 & 0.12 & 1.20 \\
\hline & 199.35 & 48.76 & 0.20 & 1.31 \\
\hline & 201.60 & 48.98 & -0.13 & 1.03 \\
\hline & 203.10 & 49.19 & 0.19 & 1.31 \\
\hline & 203.85 & 49.28 & 0.26 & 1.39 \\
\hline & 207.55 & 57.58 & -0.93 & -0.19 \\
\hline & 208.28 & 57.72 & 0.37 & 1.53 \\
\hline & 209.05 & 57.94 & 0.14 & 1.47 \\
\hline & 209.78 & 58.15 & 0.08 & 1.55 \\
\hline & 210.55 & 58.38 & 0.23 & 1.56 \\
\hline & 211.28 & 58.59 & 0.00 & 1.67 \\
\hline & 212.05 & 58.81 & 0.03 & 1.94 \\
\hline & 216.89 & 60.20 & 0.22 & 2.71 \\
\hline & 217.25 & 60.30 & 0.11 & 2.50 \\
\hline & 218.00 & 60.52 & 0.11 & 2.40 \\
\hline \multirow[t]{14}{*}{ Subbotina linaperta $(\mathrm{E})$} & 170.78 & 45.32 & 0.77 & 1.20 \\
\hline & 172.32 & 45.51 & 0.91 & 1.23 \\
\hline & 173.82 & 45.69 & 0.81 & 1.09 \\
\hline & 175.32 & 45.88 & 1.03 & 1.33 \\
\hline & 176.82 & 46.07 & 0.32 & 0.77 \\
\hline & 178.92 & 46.33 & 0.46 & 0.87 \\
\hline & 180.42 & 46.52 & 0.50 & 1.08 \\
\hline & 181.92 & 46.70 & 0.51 & 1.14 \\
\hline & 183.42 & 46.89 & 0.61 & 1.16 \\
\hline & 188.61 & 47.53 & 0.79 & 1.07 \\
\hline & 191.61 & 47.87 & 0.49 & 1.01 \\
\hline & 198.31 & 48.64 & 0.28 & 1.37 \\
\hline & 199.78 & 48.81 & 0.08 & 1.40 \\
\hline & 201.28 & 48.98 & 0.23 & 1.18 \\
\hline \multirow[t]{12}{*}{ Subbotina $\mathrm{sp}(\mathrm{E})$} & 151.41 & 40.98 & 1.02 & 1.21 \\
\hline & 152.91 & 41.12 & 1.10 & 1.48 \\
\hline & 154.41 & 41.40 & 0.99 & 1.42 \\
\hline & 155.91 & 41.86 & 0.96 & 1.13 \\
\hline & 157.41 & 42.33 & 1.15 & 1.39 \\
\hline & 158.28 & 42.60 & 1.11 & 1.62 \\
\hline & 159.62 & 43.01 & 1.02 & 1.54 \\
\hline & 159.91 & 43.10 & 1.24 & 1.41 \\
\hline & 161.12 & 43.48 & 0.82 & 1.42 \\
\hline & 162.62 & 43.94 & 0.39 & 1.31 \\
\hline & 164.12 & 44.33 & 0.53 & 1.05 \\
\hline & 169.32 & 45.12 & 0.42 & 1.00 \\
\hline \multirow[t]{3}{*}{ Subbotina pseudobulloides $(<\mathrm{F})$} & 227.50 & 64.43 & 0.96 & 1.85 \\
\hline & 228.68 & 64.77 & 0.39 & 1.80 \\
\hline & 230.20 & 65.22 & 0.89 & 1.84 \\
\hline \multirow[t]{3}{*}{ Subbotina trioculinoides $(<\mathrm{F})$} & 231.54 & 65.61 & 0.65 & 1.82 \\
\hline & 231.72 & 65.66 & 0.62 & 1.77 \\
\hline & 232.03 & 65.76 & 0.21 & 1.73 \\
\hline \multirow[t]{4}{*}{ Eoglobigerina fringa $(<\mathrm{F})$} & 232.66 & 65.94 & 0.83 & 1.48 \\
\hline & 232.66 & 65.94 & 0.83 & 1.24 \\
\hline & 233.10 & 66.07 & 1.01 & 1.25 \\
\hline & 233.10 & 66.07 & 0.11 & 1.43 \\
\hline \multirow[t]{4}{*}{ Acarinina appressocamerata (E) } & 135.39 & 39.51 & -1.16 & 2.24 \\
\hline & 137.29 & 39.64 & -1.46 & 2.26 \\
\hline & 140.08 & 39.91 & -0.91 & 2.43 \\
\hline & 143.08 & 40.20 & -0.80 & 2.73 \\
\hline A. appressocamerata (D) & 138.58 & 39.77 & -0.95 & 2.84 \\
\hline A. appressocamerata (D and E) & 141.58 & 40.05 & -1.00 & 2.79 \\
\hline Acarinina bullbrooki (E) & 202.78 & 49.15 & -0.63 & 2.72 \\
\hline & 204.28 & 49.33 & -0.85 & 2.45 \\
\hline & 205.10 & 49.50 & -0.76 & 2.57 \\
\hline
\end{tabular}

Table 2 (continued).

\begin{tabular}{lrrrr}
\hline $\begin{array}{c}\text { Species } \\
\text { (size fraction) }\end{array}$ & $\begin{array}{c}\text { Depth } \\
\text { (mbsf) }\end{array}$ & $\begin{array}{c}\text { Age } \\
\text { (Ma) }\end{array}$ & $\delta^{18} \mathrm{O}$ & $\delta^{13} \mathrm{C}$ \\
\hline Acarinina camerata (E) & 191.61 & 47.87 & -0.21 & 2.24 \\
& 198.31 & 48.64 & -0.63 & 2.25 \\
& 199.78 & 48.81 & -0.30 & 2.42 \\
Acarinina primitiva (D and E) & 162.16 & 43.80 & 0.33 & 2.60 \\
& 162.90 & 44.03 & -0.13 & 2.40 \\
& 163.66 & 44.20 & 0.05 & 1.50 \\
Acarinina wilcoxensis (E) & 169.32 & 45.12 & -0.03 & 2.18 \\
Morozovella convexa (E) & 209.52 & 58.08 & -0.41 & 3.02 \\
& 211.02 & 58.51 & -0.44 & 3.62 \\
Chiloquembelina cubensis (<F) & 121.30 & 36.26 & 1.668 & 2.133 \\
& 122.06 & 36.46 & 1.739 & 2.217 \\
& 122.80 & 36.65 & 1.636 & 1.983 \\
& 123.56 & 36.84 & 1.837 & 2.152 \\
& 125.82 & 37.63 & 1.910 & 2.411 \\
& 130.15 & 38.46 & 0.774 & 2.202 \\
Globigerinatheka index (E and F) & 130.87 & 38.60 & 0.648 & 2.201 \\
& 131.65 & 38.76 & 0.511 & 2.101 \\
& 132.37 & 38.91 & 0.512 & 2.312 \\
& 134.65 & 39.37 & 0.378 & 2.317 \\
& 136.15 & 39.54 & 0.101 & 2.254 \\
& 136.87 & 39.61 & 0.301 & 2.174 \\
& 137.65 & 39.69 & 0.507 & 2.101 \\
& 138.37 & 39.75 & 0.389 & 1.970 \\
& 139.75 & 39.88 & 0.362 & 1.839 \\
& & & &
\end{tabular}

time temperatures were about as high as those at the Paleocene/ Eocene boundary $\left(\sim 18^{\circ} \mathrm{C}\right)$. This peak appears to have lasted slightly longer $(\sim 1.0 \mathrm{~m} . \mathrm{y}$.). This temperature maximum appears to be recorded throughout the water column as at the Paleocene/Eocene boundary. Following the temperature maximum at $54.0 \mathrm{Ma}$ there is another decrease in surface-water values that is seen to mark the initiation of a long-term cooling trend that spanned the remainder of the Paleogene. The details in this cooling trend cannot be discerned in the Site 690 record because of two major hiatuses that occur between 49.0 and $46.0 \mathrm{Ma}$. and between 44.0 and $42.0 \mathrm{Ma}$ (Fig. 1; Stott and Kennett, this volume, chapter 34). Furthermore, the extinction of the acarininid group near the end of the middle Eocene precludes comparisons with the earlier Paleogene.

\section{Subbotinid Group}

The oxygen isotopic trends for the subbotinids through the Paleogene are similar to those exhibited by the acarininids (Fig. 1). Clearly exhibited is the warming through the middle and late Paleocene, the distinct excursion at the Paleocene/Eocene boundary, a slight cooling during the earliest Eocene followed by a second warm interval at $\sim 54.0 \mathrm{Ma}$, and then the initiation of a long-term cooling trend throughout the remainder of the Eocene. The isotopic values for the Subbotina species are similar to those of benthic foraminifers (Kennett and Stott, this volume). The isotopic variability is lower than that of the acarininids. This is true also during the major warm excursions at the Paleocene/Eocene boundary and at $54.0 \mathrm{Ma}$ during the early Eocene when the subbotinids exhibited a much sharper temperature change than the acarininids.

The gradient between the subbotinid and the acarininid groups averaged $0.75 \%$ during the late Paleocene. The gradient increased during the early Eocene to $1.25 \%$ and was maintained throughout the early and middle Eocene.

\section{Site 689}

\section{Subbotinid and Acarininid Groups}

Fewer data have been generated for the acarininid species from Site 689 compared with Site 690 . The focus therefore is on the subbotinid record. Much of the early Eocene is missing in a long hiatus (Fig. 2) but the record above $50.0 \mathrm{Ma}$ is well devel- 
Table 3. Planktonic foraminifer isotope data for ODP Site 690.

\begin{tabular}{|c|c|c|c|c|}
\hline $\begin{array}{c}\text { Species } \\
\text { (size fraction) }\end{array}$ & $\begin{array}{l}\text { Depth } \\
\text { (mbsf) }\end{array}$ & $\begin{array}{l}\text { Age } \\
(\mathrm{Ma})\end{array}$ & $\delta^{18} \mathrm{O}$ & $\delta^{13} \mathrm{C}$ \\
\hline \multirow[t]{18}{*}{ Subbotina angiporoides (E and $\mathrm{F}$ ) } & 93.95 & 38.57 & 1.75 & 1.07 \\
\hline & 94.70 & 38.84 & 1.14 & 1.26 \\
\hline & 95.45 & 39.11 & 1.56 & 1.24 \\
\hline & 96.20 & 39.38 & 1.68 & 1.01 \\
\hline & 96.95 & 39.58 & 1.46 & 1.20 \\
\hline & 97.70 & 39.76 & 1.57 & 1.55 \\
\hline & 99.16 & 40.11 & 1.42 & 1.54 \\
\hline & 99.16 & 40.11 & 1.15 & 1.30 \\
\hline & 99.92 & 40.29 & 1.48 & 1.51 \\
\hline & 99.92 & 40.29 & 1.63 & 1.22 \\
\hline & 99.92 & 40.29 & 1.54 & 1.18 \\
\hline & 100.68 & 40.47 & 1.77 & 1.37 \\
\hline & 100.68 & 40.47 & 1.59 & 1.62 \\
\hline & 102.16 & 40.83 & 1.53 & 1.25 \\
\hline & 104.00 & 41.27 & 1.39 & 1.13 \\
\hline & 105.49 & 41.62 & 1.25 & 1.11 \\
\hline & 105.90 & 41.77 & 1.21 & 1.35 \\
\hline & 106.66 & 41.88 & 1.05 & 1.42 \\
\hline \multirow[t]{60}{*}{ S. eocaenica (D and E) } & 101.42 & 40.65 & 1.57 & 1.39 \\
\hline & 104.40 & 41.36 & 1.18 & 1.57 \\
\hline & 107.40 & 44.69 & 0.54 & 1.17 \\
\hline & 109.60 & 44.97 & 0.55 & 1.15 \\
\hline & 110.36 & 45.06 & 0.55 & 1.16 \\
\hline & 111.10 & 45.16 & 0.42 & 1.00 \\
\hline & 111.86 & 45.25 & 0.62 & 1.31 \\
\hline & 112.60 & 45.34 & 0.59 & 1.28 \\
\hline & 114.10 & 45.53 & 0.51 & 1.32 \\
\hline & 114.86 & 45.63 & 0.46 & 1.17 \\
\hline & 115.60 & 45.72 & 0.76 & 1.37 \\
\hline & 116.36 & 45.81 & 0.53 & 1.24 \\
\hline & 117.10 & 45.91 & 0.76 & 1.40 \\
\hline & 117.86 & 46.00 & 0.48 & 1.18 \\
\hline & 119.60 & 49.30 & 0.43 & 1.41 \\
\hline & 120.36 & 49.49 & 0.28 & 1.36 \\
\hline & 121.86 & 49.86 & 0.24 & 1.43 \\
\hline & 122.60 & 50.04 & 0.26 & 1.46 \\
\hline & 124.12 & 50.42 & 0.25 & 1.52 \\
\hline & 124.88 & 50.61 & 0.24 & 1.54 \\
\hline & 126.38 & 50.98 & 0.18 & 1.18 \\
\hline & 127.12 & 51.16 & 0.07 & 1.31 \\
\hline & 130.70 & 52.05 & -0.07 & 1.31 \\
\hline & 132.20 & 52.42 & -0.12 & 1.31 \\
\hline & 132.96 & 52.61 & 0.31 & 1.69 \\
\hline & 134.46 & 53.08 & -0.59 & -0.16 \\
\hline & 135.20 & 53.31 & -0.26 & 0.55 \\
\hline & 136.70 & 53.79 & -0.20 & 0.60 \\
\hline & 137.46 & 53.96 & -0.18 & 0.66 \\
\hline & 138.16 & 54.09 & 0.00 & 0.59 \\
\hline & 138.90 & 54.23 & 0.10 & 0.69 \\
\hline & 138.90 & 54.26 & 0.46 & 1.01 \\
\hline & 138.90 & 54.26 & -0.02 & 0.67 \\
\hline & 140.40 & 54.60 & 0.07 & 0.80 \\
\hline & 141.16 & 54.74 & 0.00 & 0.74 \\
\hline & 141.16 & 54.74 & 0.42 & 0.87 \\
\hline & 142.66 & 54.92 & 0.29 & 0.98 \\
\hline & 143.40 & 55.01 & 0.09 & 0.93 \\
\hline & 143.40 & 55.01 & 0.42 & 0.91 \\
\hline & 144.16 & 55.11 & 0.46 & 1.21 \\
\hline & 145.66 & 55.26 & -0.17 & 1.26 \\
\hline & 146.40 & 55.33 & 0.04 & 0.88 \\
\hline & 147.16 & 55.41 & 0.11 & 1.24 \\
\hline & 147.86 & 55.48 & 0.32 & 1.38 \\
\hline & 149.36 & 55.62 & 0.42 & 1.49 \\
\hline & 151.60 & 55.84 & -0.12 & 1.05 \\
\hline & 152.36 & 55.92 & -0.02 & 1.13 \\
\hline & 153.10 & 55.99 & -0.01 & 1.15 \\
\hline & 153.86 & 56.07 & 0.00 & 1.38 \\
\hline & 154.60 & 56.12 & -0.31 & 1.09 \\
\hline & 155.36 & 56.18 & 0.21 & 1.30 \\
\hline & 156.10 & 56.24 & -0.24 & 1.10 \\
\hline & 156.86 & 56.29 & -0.12 & 1.12 \\
\hline & 158.30 & 56.40 & -0.20 & 1.14 \\
\hline & 158.30 & 56.40 & 0.28 & 1.37 \\
\hline & 159.06 & 56.46 & -0.23 & 1.28 \\
\hline & 159.80 & 56.52 & -0.16 & 1.27 \\
\hline & 160.56 & 56.57 & -0.46 & 1.07 \\
\hline & 161.31 & 56.63 & -0.16 & 1.10 \\
\hline & 162.80 & 56.74 & 0.10 & 1.39 \\
\hline
\end{tabular}

Table 3 (continued).

\begin{tabular}{|c|c|c|c|c|}
\hline $\begin{array}{c}\text { Species } \\
\text { (size fraction) }\end{array}$ & $\begin{array}{l}\text { Depth } \\
\text { (mbsf) }\end{array}$ & $\begin{array}{l}\text { Age } \\
(\mathrm{Ma})\end{array}$ & $\delta^{18} \mathrm{O}$ & $\delta^{13} \mathrm{C}$ \\
\hline \multirow[t]{48}{*}{ S. eocaenica (D and E) } & 163.56 & 56.80 & -0.01 & 1.15 \\
\hline & 164.30 & 56.86 & -0.27 & 1.28 \\
\hline & 165.06 & 56.91 & -0.18 & 1.23 \\
\hline & 165.80 & 56.97 & -0.09 & 1.35 \\
\hline & 167.26 & 57.08 & -0.39 & 0.87 \\
\hline & 167.99 & 57.14 & -0.46 & 0.77 \\
\hline & 168.76 & 57.19 & -0.50 & 0.37 \\
\hline & 169.49 & 57.25 & -0.73 & 0.36 \\
\hline & 170.26 & 57.31 & -1.12 & -0.58 \\
\hline & 170.99 & 57.36 & -0.08 & 1.35 \\
\hline & 173.99 & 57.59 & 0.33 & 1.65 \\
\hline & 175.30 & 57.69 & 0.11 & 2.36 \\
\hline & 176.06 & 57.75 & 0.75 & 2.16 \\
\hline & 176.80 & 57.80 & 0.22 & 2.29 \\
\hline & 178.30 & 57.92 & 0.20 & 2.53 \\
\hline & 179.06 & 57.97 & 0.35 & 2.55 \\
\hline & 179.80 & 58.03 & 0.25 & 2.31 \\
\hline & 180.66 & 58.10 & 0.23 & 2.38 \\
\hline & 181.44 & 58.15 & -0.13 & 2.10 \\
\hline & 182.16 & 58.21 & -0.02 & 2.40 \\
\hline & 182.94 & 58.27 & 0.37 & 2.73 \\
\hline & 183.66 & 58.32 & 0.20 & 2.41 \\
\hline & 184.77 & 58.41 & 0.27 & 2.28 \\
\hline & 185.56 & 58.66 & 0.49 & 2.89 \\
\hline & 187.06 & 58.76 & 0.08 & 2.70 \\
\hline & 187.80 & 58.80 & 0.26 & 3.01 \\
\hline & 189.30 & 58.90 & 0.23 & 2.65 \\
\hline & 190.06 & 58.95 & 0.24 & 2.93 \\
\hline & 190.80 & 58.99 & 0.33 & 2.63 \\
\hline & 191.56 & 59.04 & 0.27 & 2.59 \\
\hline & 194.56 & 59.23 & 0.31 & 3.18 \\
\hline & 196.06 & 59.33 & 1.64 & 3.15 \\
\hline & 196.80 & 59.37 & 0.25 & 2.73 \\
\hline & 197.56 & 59.42 & 0.91 & 2.92 \\
\hline & 198.30 & 59.46 & -0.08 & 3.11 \\
\hline & 198.56 & 59.49 & 0.30 & 3.04 \\
\hline & 200.06 & 59.58 & -0.02 & 2.97 \\
\hline & 200.80 & 59.63 & 0.23 & 3.18 \\
\hline & 203.06 & 59.77 & 0.19 & 3.12 \\
\hline & 203.80 & 59.82 & 0.25 & 3.25 \\
\hline & 204.56 & 59.87 & 0.46 & 3.30 \\
\hline & 205.30 & 59.91 & 0.46 & 2.72 \\
\hline & 206.06 & 59.96 & 0.23 & 2.54 \\
\hline & 206.80 & 60.01 & 0.58 & 2.75 \\
\hline & 207.56 & 60.06 & 0.48 & 2.83 \\
\hline & 209.80 & 60.20 & 0.49 & 2.70 \\
\hline & 210.48 & 60.24 & 0.62 & 2.63 \\
\hline & 212.08 & 60.34 & 0.38 & 2.58 \\
\hline \multirow[t]{16}{*}{ S. linaperta $(\mathrm{E})$} & 105.49 & 41.62 & 1.36 & 1.50 \\
\hline & 107.08 & 44.65 & 0.70 & 1.01 \\
\hline & 109.28 & 44.93 & 0.75 & 0.87 \\
\hline & 112.28 & 45.30 & 0.80 & 0.99 \\
\hline & 113.78 & 45.49 & 0.62 & 0.89 \\
\hline & 115.28 & 45.67 & 0.50 & 1.16 \\
\hline & 116.78 & 45.87 & 0.64 & 1.37 \\
\hline & 117.67 & 45.98 & 0.57 & 1.33 \\
\hline & 119.18 & 49.19 & 0.40 & 1.29 \\
\hline & 120.78 & 49.59 & 0.45 & 1.12 \\
\hline & 123.78 & 50.33 & 0.36 & 1.43 \\
\hline & 125.28 & 50.70 & 0.38 & 1.22 \\
\hline & 128.89 & 51.60 & 0.31 & 1.32 \\
\hline & 130.39 & 51.97 & 0.07 & 1.13 \\
\hline & 131.89 & 52.34 & 0.23 & 1.38 \\
\hline & 133.79 & 52.87 & 0.20 & 1.31 \\
\hline \multirow[t]{2}{*}{ S. triangularis (C) } & 172.19 & 57.45 & 0.25 & 1.73 \\
\hline & 207.97 & 60.08 & 0.74 & 2.93 \\
\hline \multirow[t]{12}{*}{ S. triangularis (D) } & 138.58 & 54.15 & 0.32 & 0.88 \\
\hline & 140.08 & 54.49 & -0.03 & 0.63 \\
\hline & 148.30 & 55.52 & 0.46 & 1.26 \\
\hline & 149.80 & 55.67 & 0.35 & 1.38 \\
\hline & 151.30 & 55.81 & 0.32 & 1.26 \\
\hline & 152.80 & 55.96 & 0.39 & 1.21 \\
\hline & 154.30 & 56.11 & 0.35 & 1.31 \\
\hline & 156.67 & 56.28 & 0.16 & 1.30 \\
\hline & 159.49 & 56.49 & 0.12 & 1.41 \\
\hline & 160.99 & 56.61 & 0.14 & 1.35 \\
\hline & 162.49 & 56.72 & -0.17 & 1.36 \\
\hline & 166.99 & 57.06 & -0.07 & 1.29 \\
\hline
\end{tabular}


Table 3 (continued).

\begin{tabular}{|c|c|c|c|c|}
\hline $\begin{array}{c}\text { Species } \\
\text { (size fraction) }\end{array}$ & $\begin{array}{l}\text { Depth } \\
\text { (mbsf) }\end{array}$ & $\begin{array}{l}\text { Age } \\
(\mathrm{Ma})\end{array}$ & $\delta^{18} \mathrm{O}$ & $\delta^{13} \mathrm{C}$ \\
\hline \multirow[t]{3}{*}{$S$, triangularis (D) } & 167.69 & 57.11 & 0.07 & 0.78 \\
\hline & 170.69 & 57.34 & 0.04 & 1.87 \\
\hline & 207.97 & 60.08 & 0.98 & 3.16 \\
\hline \multirow[t]{27}{*}{ S. triangularis $(\mathrm{E})$} & 135.39 & 53.37 & -0.05 & 0.75 \\
\hline & 140.08 & 54.49 & 0.28 & 0.64 \\
\hline & 144.58 & 55.16 & 0.66 & 1.11 \\
\hline & 146.08 & 55.30 & 0.52 & 1.36 \\
\hline & 148.30 & 55.52 & 0.52 & 1.33 \\
\hline & 151.30 & 55.81 & 0.22 & 1.12 \\
\hline & 152.80 & 55.96 & 0.23 & 1.14 \\
\hline & 155.80 & 56.21 & 0.25 & 1.38 \\
\hline & 156.67 & 56.28 & 0.16 & 1.34 \\
\hline & 160.99 & 56.61 & 0.12 & 1.37 \\
\hline & 163.99 & 56.83 & -0.01 & 1.40 \\
\hline & 169.19 & 57.23 & -0.41 & 0.60 \\
\hline & 170.69 & 57.34 & 0.11 & 1.65 \\
\hline & 172.19 & 57.45 & 0.36 & 1.90 \\
\hline & 173.69 & 57.57 & 0.20 & 1.52 \\
\hline & 173.69 & 57.57 & 0.41 & 1.86 \\
\hline & 175.07 & 57.67 & -0.12 & 2.10 \\
\hline & 176.57 & 57.79 & 0.17 & 1.93 \\
\hline & 178.07 & 57.90 & 0.40 & 2.34 \\
\hline & 179.57 & 58.01 & 0.41 & 2.49 \\
\hline & 181.08 & 58.13 & -0.09 & 2.14 \\
\hline & 182.58 & 58.24 & 0.32 & 2.69 \\
\hline & 183.97 & 58.35 & 0.23 & 2.33 \\
\hline & 193.51 & 59.17 & 0.20 & 3.02 \\
\hline & 195.01 & 59.26 & 0.40 & 3.03 \\
\hline & 196.51 & 59.36 & 0.24 & 3.18 \\
\hline & 207.97 & 60.08 & 0.80 & 3.02 \\
\hline \multirow[t]{2}{*}{ Acarinina appressocamerata (D) } & 138.58 & 54.15 & -0.95 & 2.84 \\
\hline & 141.58 & 54.79 & -1.00 & 2.79 \\
\hline \multirow[t]{5}{*}{ A. appressocamerata $(\mathrm{E})$} & 135.39 & 53.37 & -1.20 & 2.24 \\
\hline & 137.29 & 53.92 & -1.50 & 2.26 \\
\hline & 140.08 & 54.49 & -0.90 & 2.43 \\
\hline & 141.58 & 54.79 & -1.00 & 2.79 \\
\hline & 143.08 & 54.97 & -0.80 & 2.73 \\
\hline \multirow[t]{7}{*}{ A. camerata $(\mathrm{E})$} & 116.78 & 45.87 & 0.00 & 2.90 \\
\hline & 117.67 & 45.98 & -0.25 & 2.69 \\
\hline & 119.18 & 49.19 & -0.11 & 2.63 \\
\hline & 120.78 & 49.59 & -0.35 & 2.22 \\
\hline & 123.78 & 50.33 & -0.09 & 2.74 \\
\hline & 125.28 & 50.70 & -0.39 & 2.79 \\
\hline & 128.89 & 51.60 & -0.47 & 2.62 \\
\hline \multirow[t]{13}{*}{ A. primitiva (D) } & 107.36 & 44.69 & 0.15 & 1.98 \\
\hline & 108.50 & 44.87 & 0.12 & 1.94 \\
\hline & 108.86 & 44.88 & -0.29 & 2.02 \\
\hline & 108.86 & 44.88 & -0.49 & 2.23 \\
\hline & 113.36 & 45.44 & -0.07 & 2.08 \\
\hline & 118.86 & 49.11 & 0.14 & 1.93 \\
\hline & 123.36 & 50.23 & -0.98 & 2.77 \\
\hline & 123.36 & 50.23 & -0.83 & 2.70 \\
\hline & 126.38 & 50.98 & -0.17 & 1.90 \\
\hline & 128.46 & 51.49 & -0.67 & 2.73 \\
\hline & 129.20 & 51.68 & -0.90 & 2.68 \\
\hline & 129.96 & 51.87 & -0.92 & 2.23 \\
\hline & 131.46 & 52.24 & -0.44 & 2.29 \\
\hline A. pseudotopilensis (C) & 115.28 & 45.68 & 0.47 & 1.99 \\
\hline A. pseudotopilensis (D) & 115.28 & 45.68 & 0.37 & 1.68 \\
\hline \multirow[t]{12}{*}{ A. pseudotopilensis (E) } & 107.08 & 44.65 & 0.25 & 1.45 \\
\hline & 109.28 & 44.93 & -0.09 & 1.96 \\
\hline & 112.28 & 45.30 & 0.19 & 1.72 \\
\hline & 115.28 & 45.68 & 0.25 & 1.48 \\
\hline & 116.78 & 45.87 & 0.41 & 1.73 \\
\hline & 117.67 & 45.98 & 0.20 & 1.57 \\
\hline & 126.78 & 51.08 & -0.37 & 1.94 \\
\hline & 127.67 & 51.30 & -0.82 & 2.73 \\
\hline & 130.39 & 51.97 & -0.11 & 1.59 \\
\hline & 131.89 & 52.34 & -1.01 & 2.83 \\
\hline & 133.79 & 52.87 & -0.82 & 3.00 \\
\hline & 143.08 & 54.97 & 0.18 & 1.00 \\
\hline \multirow[t]{6}{*}{ A. soldadoensis (D) } & 147.86 & 55.48 & -0.40 & 3.06 \\
\hline & 153.86 & 56.07 & -0.92 & 3.23 \\
\hline & 154.60 & 56.14 & -1.01 & 3.33 \\
\hline & 164.30 & 56.86 & -2.62 & 1.89 \\
\hline & 169.49 & 57.25 & -1.76 & 1.62 \\
\hline & 170.26 & 57.31 & -1.99 & 0.66 \\
\hline
\end{tabular}

Table 3 (continued).

\begin{tabular}{|c|c|c|c|c|}
\hline $\begin{array}{c}\text { Species } \\
\text { (size fraction) }\end{array}$ & $\begin{array}{l}\text { Depth } \\
\text { (mbsf) }\end{array}$ & $\begin{array}{l}\text { Age } \\
(\mathrm{Ma})\end{array}$ & $\delta^{18} \mathrm{O}$ & $\delta^{13} \mathrm{C}$ \\
\hline \multirow[t]{2}{*}{ A. soldadoensis $(\mathrm{E})$} & 165.06 & 56.91 & -2.20 & 1.90 \\
\hline & 170.26 & 57.31 & -1.90 & 0.36 \\
\hline \multirow[t]{10}{*}{ Muricoglobigerina mckannai (D) } & 147.86 & 55.48 & -2.05 & 1.82 \\
\hline & 154.30 & 56.11 & -1.06 & 3.07 \\
\hline & 159.49 & 56.49 & -0.92 & 3.31 \\
\hline & 160.99 & 56.61 & -0.98 & 3.26 \\
\hline & 162.49 & 56.72 & -0.88 & 3.06 \\
\hline & 163.99 & 56.83 & -0.99 & 3.01 \\
\hline & 165.49 & 56.95 & -1.08 & 3.24 \\
\hline & 170.26 & 57.31 & -2.15 & -0.58 \\
\hline & 170.69 & 57.34 & -1.33 & 0.04 \\
\hline & 172.19 & 57.45 & -0.29 & 2.90 \\
\hline \multirow{22}{*}{ M. mckannai (E) } & 147.86 & 55.48 & -1.34 & 2.05 \\
\hline & 148.30 & 55.52 & -0.65 & 3.07 \\
\hline & 157.99 & 56.38 & -0.94 & 3.11 \\
\hline & 160.99 & 56.61 & -0.85 & 3.09 \\
\hline & 162.49 & 56.72 & -0.88 & 3.06 \\
\hline & 163.99 & 56.83 & -0.86 & 2.82 \\
\hline & 166.99 & 57.05 & -0.62 & 2.12 \\
\hline & 169.19 & 57.23 & -1.29 & 1.98 \\
\hline & 170.26 & 57.31 & -1.56 & -0.33 \\
\hline & 170.69 & 57.34 & -0.52 & 2.15 \\
\hline & 170.99 & 57.36 & -0.33 & 3.02 \\
\hline & 171.76 & 57.42 & -1.44 & 1.98 \\
\hline & 172.19 & 57.45 & -0.29 & 2.90 \\
\hline & 173.26 & 57.54 & -1.00 & 2.86 \\
\hline & 173.69 & 57.57 & -0.74 & 6.51 \\
\hline & 188.98 & 58.88 & -0.38 & 3.53 \\
\hline & 190.48 & 58.97 & -0.09 & 4.54 \\
\hline & 192.01 & 59.07 & -0.39 & 4.38 \\
\hline & 198.78 & 59.50 & -0.30 & 4.78 \\
\hline & 200.29 & 59.60 & -0.30 & 5.04 \\
\hline & 201.79 & 59.69 & -0.30 & 5.12 \\
\hline & 203.29 & 59.78 & -0.22 & 4.96 \\
\hline M. mckannai (F) & 170.99 & 57.36 & -0.60 & 2.88 \\
\hline \multirow{4}{*}{ Eoglobigerina fringa $(<\mathrm{F})$} & 247.69 & 66.31 & 0.82 & 2.47 \\
\hline & 247.69 & 66.31 & 1.13 & 2.42 \\
\hline & 247.76 & 66.36 & 0.78 & 2.31 \\
\hline & 247.76 & 66.36 & 0.65 & 2.46 \\
\hline \multirow{9}{*}{ Subbotina pseudobulloides $(<\mathrm{F})$} & 236.58 & 64.71 & 0.57 & 2.05 \\
\hline & 242.90 & 65.53 & 0.50 & 1.99 \\
\hline & 243.83 & 65.65 & 0.54 & 2.07 \\
\hline & 244.64 & 65.76 & 0.49 & 2.02 \\
\hline & 245.35 & 65.85 & 0.59 & 2.22 \\
\hline & 245.61 & 65.88 & 0.61 & 1.93 \\
\hline & 246.17 & 65.96 & 0.68 & 1.98 \\
\hline & 246.86 & 66.05 & 0.45 & 1.68 \\
\hline & 247.24 & 66.10 & 0.61 & 1.93 \\
\hline Subbotina triloculinoides $(<\mathrm{F})$ & 243.83 & 65.65 & 0.59 & 2.01 \\
\hline
\end{tabular}

oped at this site. This is important because this portion of the record is largely missing at Site 690.

The sparse data for the Paleocene also exhibit the warming trend clearly illustrated at Site 690, with temperatures beginning at about $9^{\circ} \mathrm{C}$ in the early Paleocene and increasing to $12^{\circ} \mathrm{C}$ in the late Paleocene. This warming trend was punctuated by a sharp temperature maximum at the Paleocene/Eocene boundary as recorded in the sparse Acarinina and Subbotina records (Fig. 2).

Following the major hiatus in the early Eocene the subbotinid record exhibits a general cooling between $\sim 50.0$ and $34.0 \mathrm{Ma}$. Much of this cooling trend occurs within three major steps. These steps occur at $43.0 \mathrm{Ma}(\sim 0.5 \%), 40.0 \mathrm{Ma}$, at the middle/ late Eocene boundary $(0.5 \%)$ and at the Eocene/Oligocene boundary ( $\sim 36.0 \mathrm{Ma} ; 1.0 \%)$. Each step represents a cooling of at least $3.0^{\circ} \mathrm{C}$. There is a short reversal in this long-term cooling trend in the early Oligocene between $34.0 \mathrm{Ma}$ and $32.0 \mathrm{Ma}$ that resulted in a $3^{\circ} \mathrm{C}$ warming of the surface waters. 


\section{$\delta^{18} \mathrm{O} \%$}

Age $\mathrm{Ma}-2$

$-1$

0

1

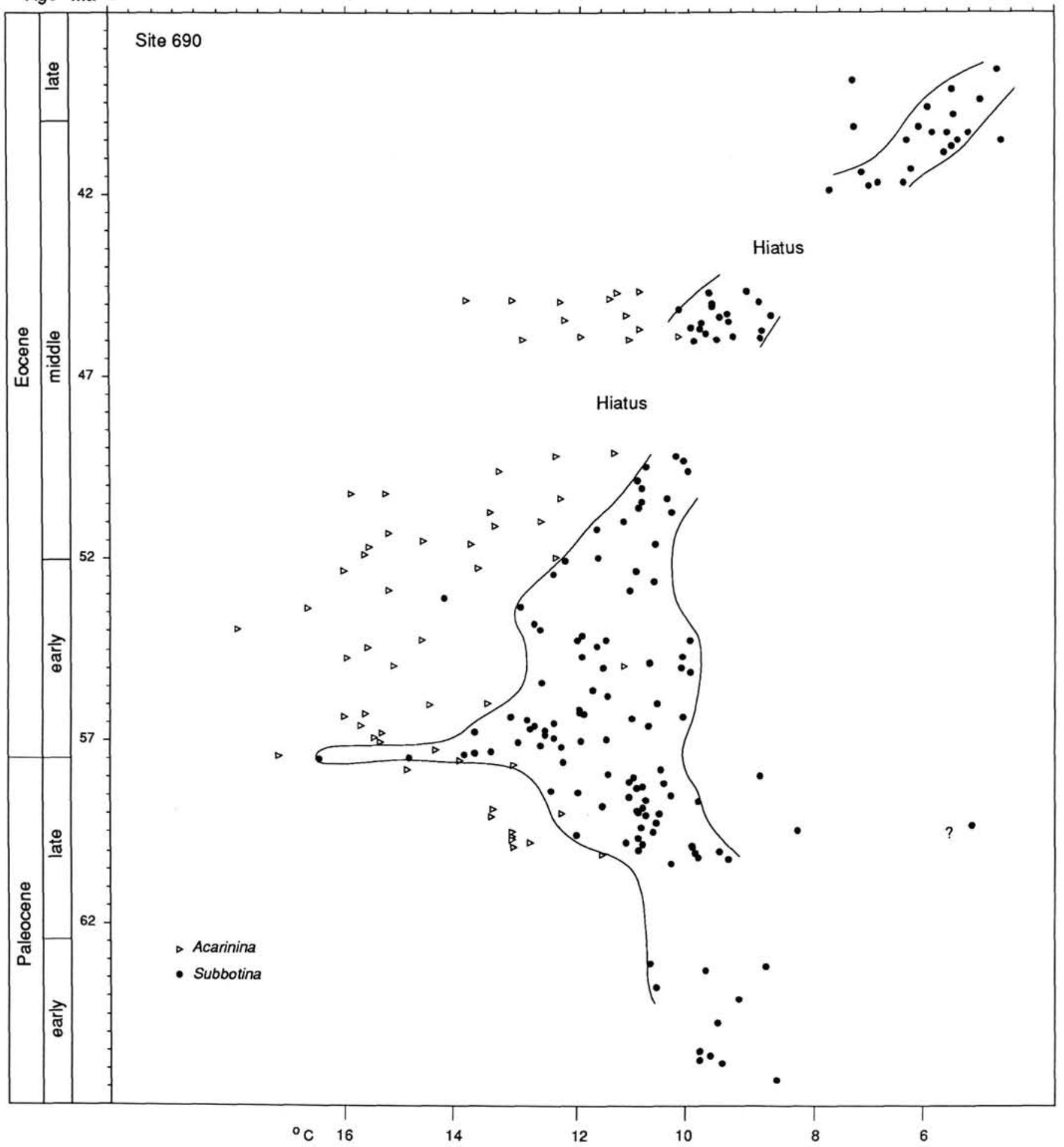

Figure 1. Oxygen isotopic composition of subbotinid and acarininid species of Site 690. The taxonomy of planktonic foraminifers is discussed in Stott and Kennett (this volume, chapter 34). The age model is based on biostratigraphic correlation and magnetostratigraphic correlations to the standard chronostratigraphic time scale of Berggren et al. (1985) using the planktonic foraminifer biostratigraphy (Stott and Kennett, this volume, chapter 34) and magnetostratigraphy of Site 690 (Spieß, this volume). Linear interpolation was carried out between chron boundaries. The temperature scale is based upon the assumption of no significant ice-sheet prior to the early Oligocene and does not account for variation in surface-water salinity during the Paleogene. The temperature equation is that of O'Neil et al. (1969) with considerations discussed by Shackleton (1974). The lines are used to encompass the range of isotopic variability and to highlight the patterns of change between the groups. Note the sharp negative excursion at the Paleocene/Eocene boundary and the low $\delta^{18} \mathrm{O}$ values for the acarininids in the early Eocene. 

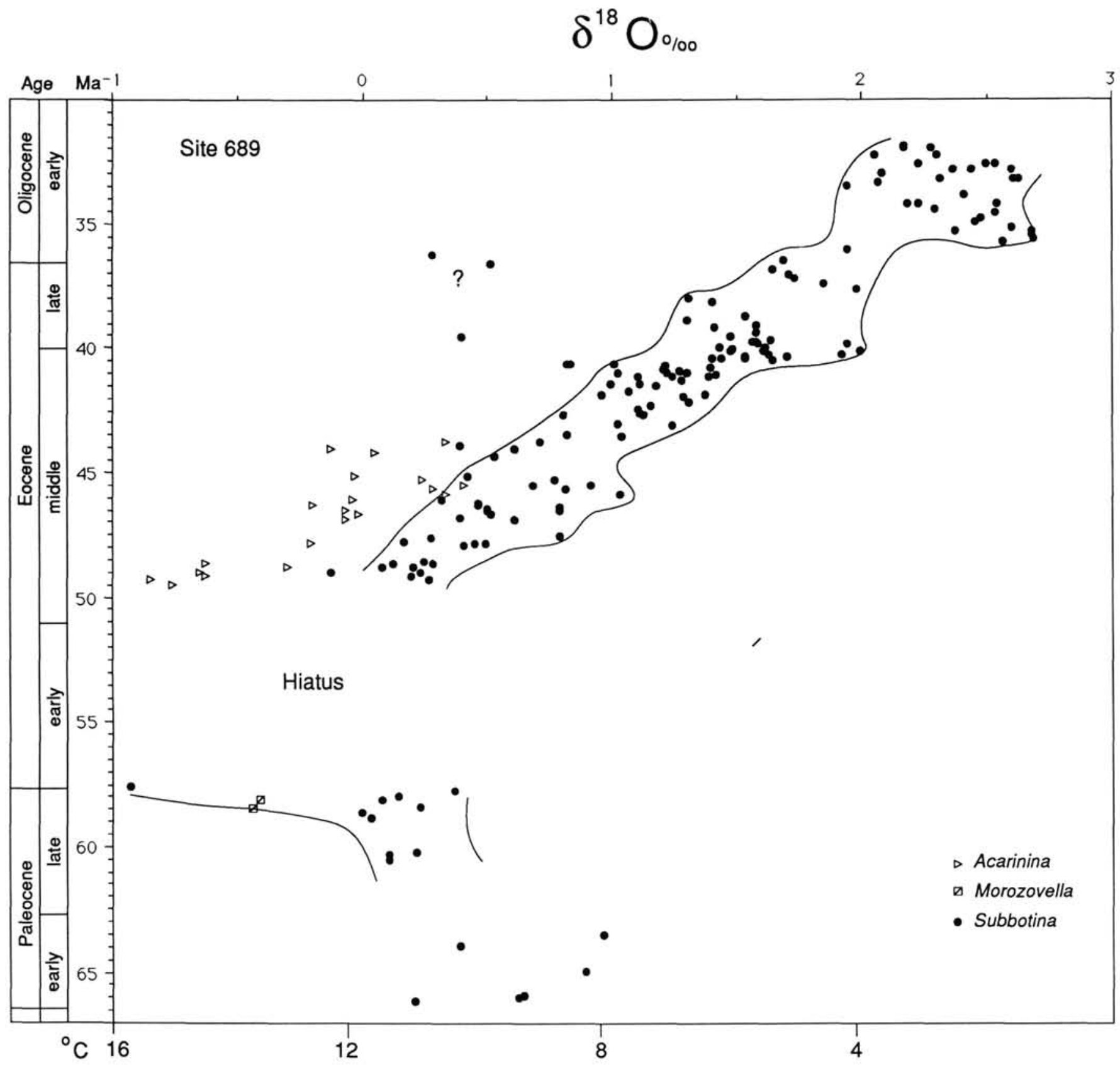

Figure 2. Oxygen isotopic composition of subbotinids, morozovellids and acarininids of Site 689. See Figure 1 for explanation. Note the hiatus that spans the early Eocene.

\section{Carbon Isotopes}

\section{Site 690}

\section{Acarininid Group}

The carbon isotopic composition of the acarininids is consistently higher than those of the subbotinid group (Fig. 3). The stratigraphy is characterized by several distinctive features. The record for the acarininid carbon isotopic stratigraphy begins in the late Paleocene when the values show an increase from $\sim 4.0 \%$ at $\sim 60.0 \mathrm{Ma}$ to $5.0 \% 0$ at $59.0 \mathrm{Ma}$. These are the highest $\delta^{13} \mathrm{C}$ values recorded in the late Phanerozoic and they persisted for
$<1.0$ m.y. After 59.0 Ma the values for the acarininid group began to decrease rapidly during the latest Paleocene, reaching values of $2.8 \%$ by $\sim 57.5$ Ma near the Paleocene/Eocene boundary (Fig. 3). This trend toward decreased $\delta^{13} \mathrm{C}$ values is well documented in the marine record (Shackleton and Kennett, 1975; Shackleton et al., 1984; Miller et al., 1987). In association with this $\delta^{13} \mathrm{C}$ shift there is a distinctive excursion in the $\delta^{13} \mathrm{C}$ values to about $2.0 \%$ in the acarininid data. This $\delta^{13} \mathrm{C}$ excursion is coincident with that observed in the $\delta^{18} \mathrm{O}$ record near the Paleocene/Eocene boundary and appears to be similar in duration, lasting $\sim 200$ k.y. The spike terminated with a return of $\delta^{13} \mathrm{C}$ values to about $3.0 \%$ for the acarininids. During the remainder 


\section{$\delta^{13} \mathrm{C}_{\%}$}

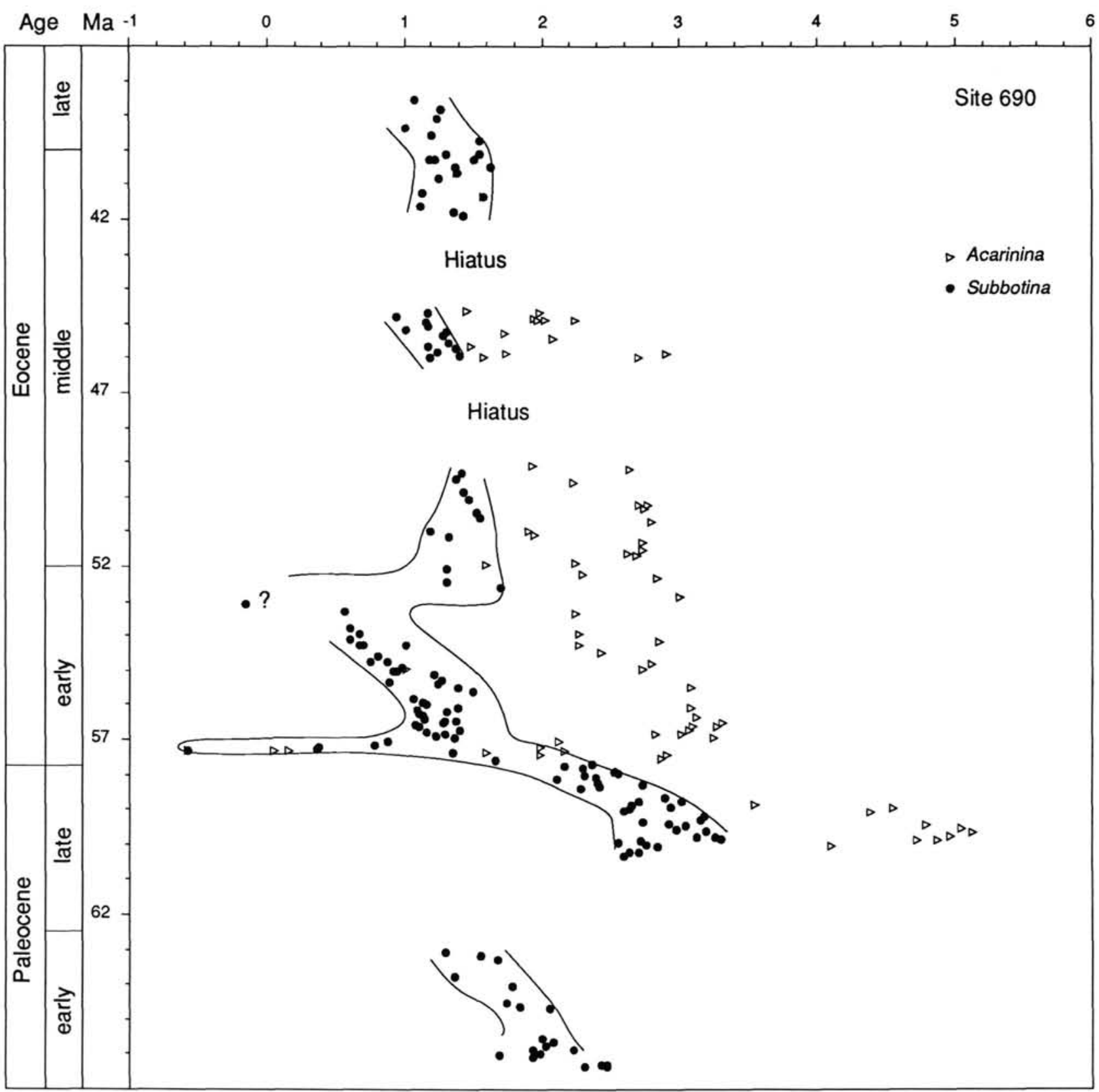

Figure 3. Carbon isotopic composition of planktonic foraminifers of ODP Site 690. See Figure 1 for explanation. Note the pattern of increasing values in the late Paleocene and the sharp negative shift between the late Paleocene and the early Eocene. Superimposed on the negative shift is a sharp excursion at the Paleocene/Eocene boundary in association with the negative oxygen isotopic excursion. The $\delta^{13} \mathrm{C}$ gradient between acarininids and subbotinids appears to be reduced briefly during this excursion. Note the well-developed $\delta^{13} \mathrm{C}$ gradient between acarininids (summer dwellers) and subbotinids.

of the early Eocene, until $\sim 45.0 \mathrm{Ma}, \delta^{13} \mathrm{C}$ values averaged about $2.7 \%$, apart from a distinctive decrease (to $\sim 2.0 \%$ ) centered at 54.0 Ma (Fig. 3).

\section{Subbotinid Group}

The carbon isotopic trends for the subbotinids during the $\mathrm{Pa}$ leogene parallel those of the acarininids, although values were lower. The earliest Paleocene $\delta^{13} \mathrm{C}$ values immediately following the Cretaceous/Paleogene boundary were relatively high $(\sim 2.5 \%)$ and underwent a uniform decrease during the early Paleocene, reaching values of about $1.0 \%$ by the middle Paleocene $(\sim 63.0$ Ma) (Fig. 3). The planktonic isotopic record is incomplete between 63.0 and $60.5 \mathrm{Ma}$. The differences in values across this interval indicate that $\delta^{13} \mathrm{C}$ increased markedly during this time to reach the highest values of the late Phanerozoic at 59.0 Ma. This $\delta^{13} \mathrm{C}$ increase is also exhibited within the acarininid records. The carbon isotopic trends that followed during the remainder of the early and middle Eocene are similar to those exhibited by 


\section{$\delta^{13} \mathrm{C}_{100}$}

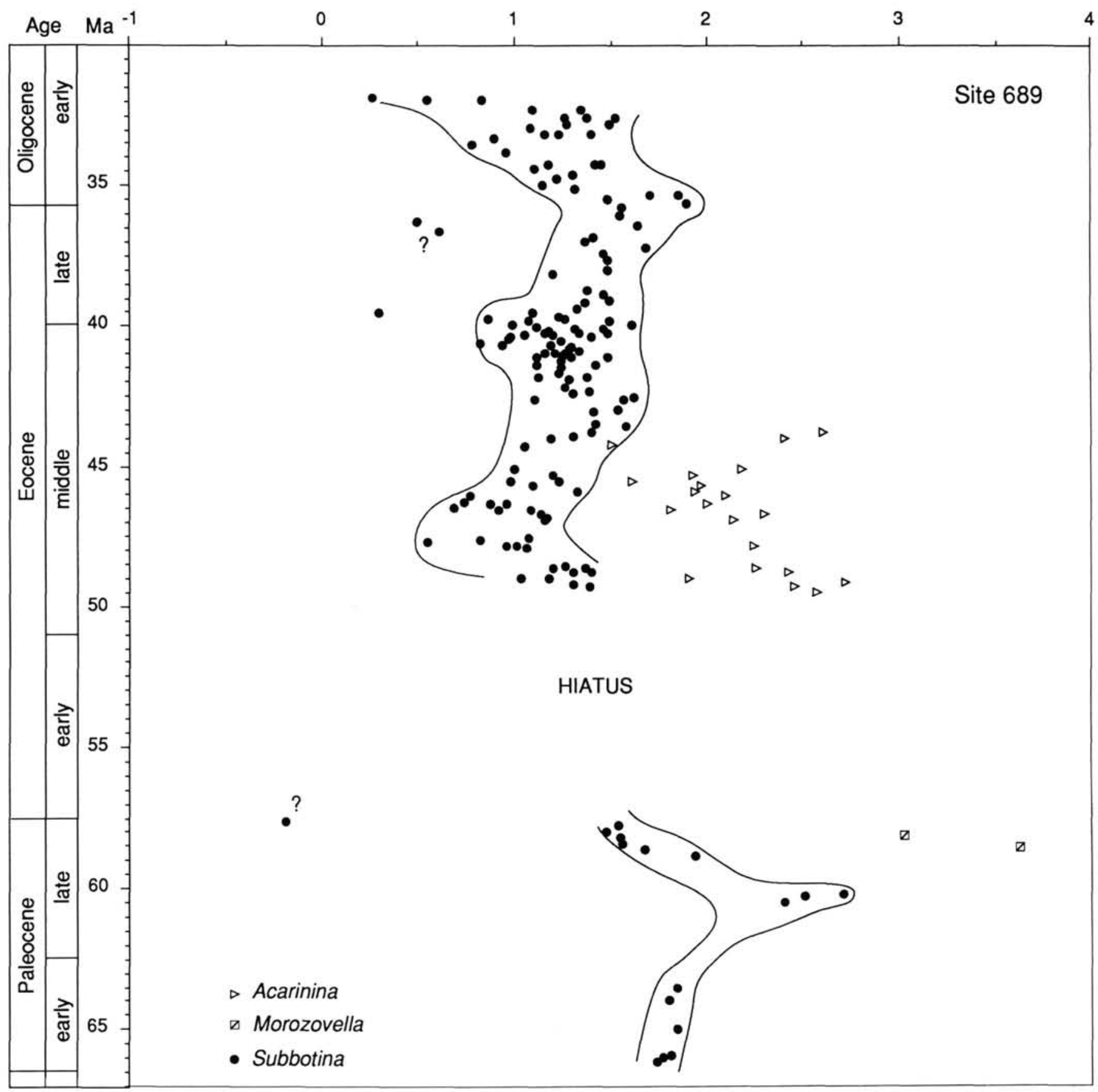

Figure 4. Carbon isotopic composition of planktonic foraminifers of ODP Site 689. See Figure 1 for explanation. Note that the sharp excursion at the Paleocene/Eocene boundary is evident also in this site. The patterns of change are similar to those of Site 690 but note that the middle and late Eocene are better represented in this site.

the acarininids. Clearly shown is the distinct carbon isotopic shift toward lower values $(\sim 1.0 \%)$ at the end of the Paleocene; the distinctive negative excursion (to $\sim-1.0 \%$ ) near the Paleocene/Eocene boundary. The remainder of the early and middle Eocene was marked by little change in $\delta^{13} \mathrm{C}$ in the subbotinids with values averaging $\sim 1.0 \%$ throughout the interval.

\section{Site 689}

\section{Subbotinid and Acarininid Group}

The sparse acarininid data add little to observations for Site 690 . The subbotinid data exhibits high values $(-2.0 \%)$ during the early Paleocene. Beginning at about $65 \mathrm{Ma}$ the $\delta^{13} \mathrm{C}$ record 
exhibits a trend toward increasing values that continued through most of the late Paleocene when values reached $\sim 2.7 \%$ by about $60 \mathrm{Ma}$ (Fig. 4). This is followed by the carbon isotopic shift at the end of the Paleocene during which time values decreased sharply to about $1.5 \%$, similar to those observed for Site 690. The large and distinctive negative excursion in $\delta^{13} \mathrm{C}$ at the Paleocene/Eocene boundary is less well constrained in this site compared to Site 690 . Nonetheless, one sample at the Paleocene/Eocene boundary does exhibit a value of $-0.25 \%$, similar to that observed for Site 690 (Fig. 4).

A hiatus has removed the record above the Paleocene/Eocene boundary to the early middle Eocene $(\sim 49.0 \mathrm{Ma})$. Following this major hiatus the subbotinid record exhibits a general increase in values from about $0.75 \% 0$ at $48.0 \mathrm{Ma}$ to about $1.5 \% 0$ at the Eocene/Oligocene boundary $(\sim 36.6 \mathrm{Ma})$. This trend toward increasing $\delta^{13} \mathrm{C}$ values did not proceed uniformly. There is a small but distinct reversal in $\delta^{13} \mathrm{C}$ values centered at about 40.0 $\mathrm{Ma}$, the boundary between the middle and late Eocene. During the early Oligocene, between 36.0 and $32.0 \mathrm{Ma}, \delta^{13} \mathrm{C}$ values became progressively lower reaching minimum values of about $0.25 \%$ by the middle Oligocene. Any changes in the $\delta^{13} \mathrm{C}$ gradient between the acarininid and the subbotinid group cannot be distinguished with the limited data.

\section{DISCUSSION}

\section{Climatic Evolution}

\section{Paleocene-early Eocene: The Climax of Cenozoic Warmth}

Data presented elsewhere in this volume (Stott and Kennett, this volume, chapter 47) indicate that, beginning about 200 k.y. prior to the $\mathrm{K} / \mathrm{T}$ boundary, surface-water temperatures in the Antarctic began to cool. Surface-water temperatures inferred from planktonic foraminifer $\delta^{18} \mathrm{O}$ values were apparently reduced by $\sim 3^{\circ} \mathrm{C}$. The data from Sites 689 and 690 indicate that surface-water temperatures were $\sim 9^{\circ}-10^{\circ} \mathrm{C}$ following the $\mathrm{K} / \mathrm{T}$ boundary. Surface-water temperatures at lower latitudes recorded in DSDP Site 577 (Zachos et al., 1989) were slightly warmer, ranging from $\sim 12^{\circ} \mathrm{C}$ in the earliest Paleogene $(\sim 66.4-66.0 \mathrm{Ma})$ to $\sim 15^{\circ} \mathrm{C}$ in the middle Paleocene $(63.0 \mathrm{Ma})$. This comparison suggests that the average meridional temperature gradient between the tropical Pacific and the Antarctic was $3^{\circ}-5^{\circ} \mathrm{C}$ in the early Paleogene. Data from middle latitude South Atlantic DSDP Site 527 (Shackleton et al., 1984) indicate that surface-water temperatures at those latitudes were similar to those in the Antarctic Ocean. It appears therefore, that most of the meridional temperature gradient occurred between the middle and low latitudes during the early Paleocene.

Beginning at $\sim 63.0 \mathrm{Ma}$, near the boundary between the early and late Paleocene, surface-water temperatures in the Antarctic began to warm. This warming trend, that continued through the late Paleocene, is recorded throughout the oceans and is taken to represent the initiation of a broad warming trend that spanned the late Paleocene and early Eocene. The sharp peak in this warming trend that occurred at the Paleocene/Eocene boundary was associated with the extinction of many deep-sea benthic foraminifer species (Thomas, this volume). The number of benthic species eliminated at this extinction event appears to be $\sim 30-35$ in the Antarctic, approximately half of the number of species that existed during the Paleocene (Thomas, this volume). The extinction also appears to have eliminated a greater number of epifaunal than infaunal species, an observation that may be important in understanding the cause of the extinctions if intermediate waters were for some reason suddenly depleted in oxygen and/or became significantly warmer (Thomas, this volume). The maximum temperature reached in the surface waters of the Antarctic during the Paleocene/Eocene boundary transition was $\sim 17^{\circ}-18^{\circ} \mathrm{C}$ (Fig. 5). Oxygen isotopic temperatures for benthic foraminifers living at intermediate water depths also record this warm spike (Kennett and Stott, this volume). This implies that the entire Antarctic water column became warmer or that there was a sudden change in the isotopic composition of the oceans. Increased mixing does not appear to be a viable explanation for this reduced stratification because this cannot explain the overall higher oxygen isotopic temperatures of both planktonic and benthic foraminifers. On the other hand, it is possible that the production of cool intermediate waters at high southern latitudes was turned off briefly at the Paleocene/Eocene boundary. This could have occurred in response to an infusion of warm waters from lower latitudes. Such waters, being older and warmer, would be lower in dissolved oxygen and would also have higher nutrient concentrations and hence lower $\delta^{13} \mathrm{C}$. Kennett and Stott (this volume) have suggested that warm waters from lower latitudes contributed to the Antarctic water masses through the $\mathrm{Pa}$ leogene. These waters occur in the Antarctic as intermediate and/or deep water rather than surface waters. Woodruff and Savin (1989) have also suggested that warm saline water formed at low latitudes greatly influenced deep-water circulation during the early Neogene. Throughout the Paleogene and early Neogene however, the Antarctic apparently remained a source of cool intermediate water and this implies that there were multiple sources of deep and intermediate water during much of the early and middle Cenozoic. The Paleocene/Eocene boundary appears to represent a short term termination of the high latitude source of cool intermediate waters. The latest Paleocene to earliest Eocene is the only time in the Antarctic biostratigraphic record when Morozovella species were a significant part of the planktonic foraminifer assemblages (Stott and Kennett, this volume, chapter 34). This represents supporting evidence for a climatic peak in the Antarctic at that time. The isotopic temperatures recorded in morozovellid species at the Paleocene/Eocene boundary are essentially the same at tropical Pacific Site 577 and Maud Rise. Both planktonic groups were probably surfacedwelling forms since they both record the lowest $\delta^{18} \mathrm{O}$ values (Fig. 5). This implies that the temperature gradient between tropical Site 577 and the Antarctic was severely reduced or briefly eliminated. At the same time there was a decrease in eolian sediment grain size in tropical Pacific deep sequences (Miller et al., 1987), an observation that itself supports the idea that meridional temperature gradients were reduced, thereby reducing zonal wind strengths.

Acarininid $\delta^{18} \mathrm{O}$ values remained relatively high during the early Eocene while subbotinids became isotopically more positive (cooler values), returning to values similar to those of the late Paleocene. Maximum temperatures recorded by the acarininids during the early Eocene were between $15^{\circ}$ and $17^{\circ} \mathrm{C}$ while maximum values exhibited by the subbotinids were between $12^{\circ}$ and $14^{\circ} \mathrm{C}$. The minimum temperature recorded by subbotinids was about $9^{\circ} \mathrm{C}$. Therefore, the total range of planktonic foraminifer temperatures were $\sim 9^{\circ}-17^{\circ} \mathrm{C}$. This range of variability is greater than that recorded for the Paleocene and may represent a broader distribution of water column habitats during the Eocene (i.e., greater temperature stratification) compared to the Paleocene. Alternatively the higher oxygen isotope variability may reflect greater seasonal temperature contrasts. In the latter case, acarininids would have inhabited warmer summer surface waters and subbotinids would have inhabited cooler surface waters during the winter and spring.

We assume that minimum temperatures expressed by subbotinid and benthic foraminifers reflect minimum temperatures recorded during the austral year. If the temperatures of intermediate waters recorded in the benthic foraminifers reflect an Antarctic source they must represent temperatures of surface waters during the colder austral winter when surface waters became 


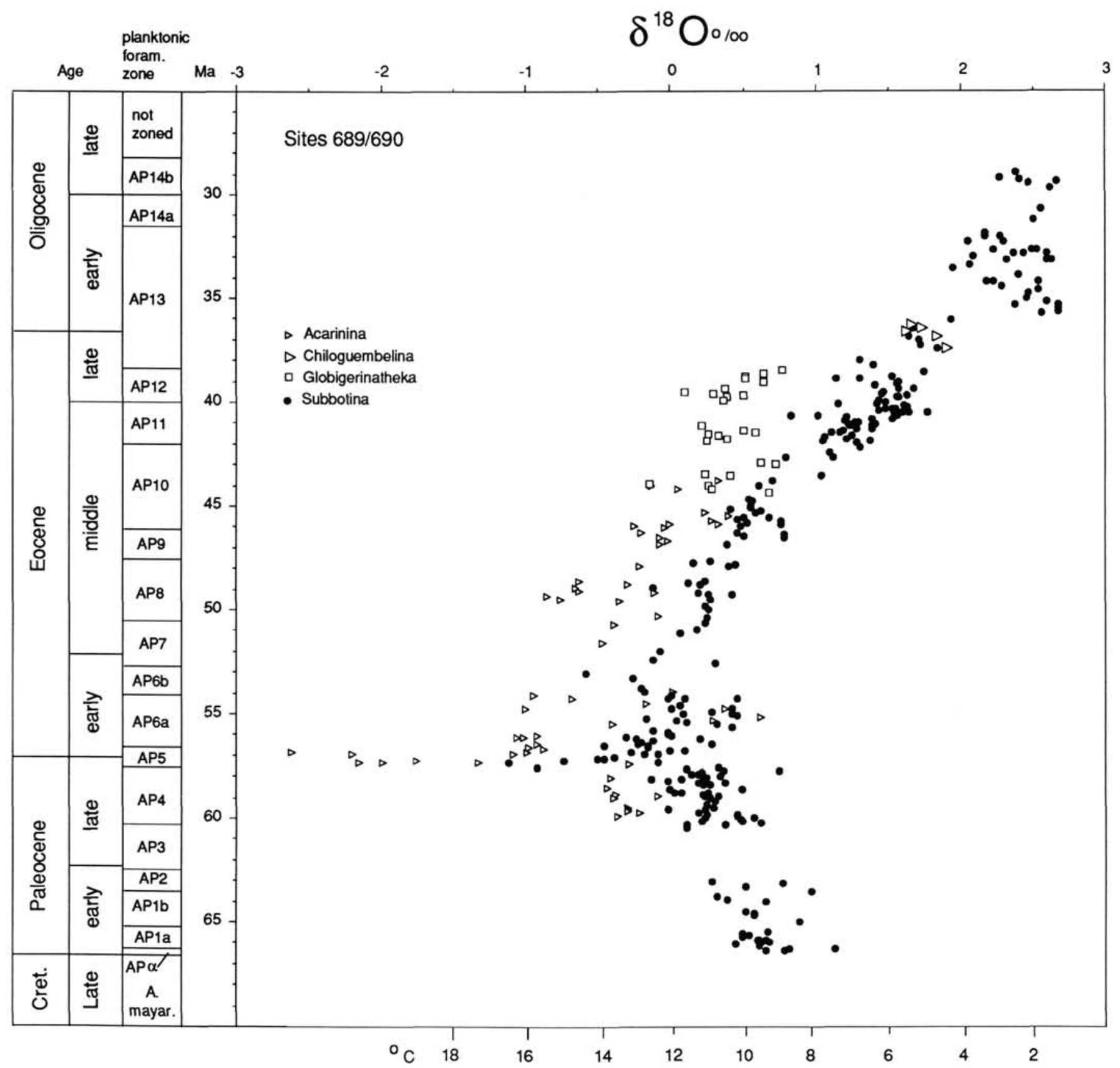

Figure 5. Composite oxygen isotopic record of planktonic foraminifers (including Chiloquembelina cubensis and Globigerinatheka index) for ODP Sites 689 and 690 . See Figure 1 for explanation.

cold enough to sink to intermediate water depths. Because the subbotinids exhibit isotopic temperatures that are similar to, but slightly warmer than, benthic foraminifers, and yet exhibit greater intrasample isotopic variability, they probably lived for a longer period of the year than the acarininids. This is also suggested by the much higher percentage of subbotinid specimens in the samples compared to other species, an observation that indicates greater production and flux of subbotinids than acarininids. Subbotinid isotopic values (based on samples consisting of 30 40 specimens) is expected to reflect a seasonally averaged signal composed of some fraction of summer, spring, autumn, and possibly winter conditions. If they were truly deeper-dwelling forms, preferring habitats below the thermocline, they would be expected to exhibit less isotopic variability because of more uniform temperatures at depth.

Based on the minimum isotopic temperatures of subbotinids and benthic foraminifers it appears that the winter sea-surface temperatures were never colder than $\sim 8^{\circ}-9^{\circ} \mathrm{C}$ during the Paleocene and early Eocene. Minimum values for the subbotinids changed only twice during the late Paleocene and early Eocene; at the Paleocene/Eocene boundary, as discussed above, and during the late early Eocene between $\sim 52.0$ and $53.0 \mathrm{Ma}$. The minimum values throughout most of the early Paleogene remained $\sim 9^{\circ}-10^{\circ} \mathrm{C}$. If it is assumed that there was no significant ice accumulation to influence the oceanic isotopic composition (Barker, Kennett, et al., 1988), then the paleotemperatures de- 
rived from the benthic foraminifers and subbotinids, taken to represent approximate winter sea-surface temperatures, precludes the existence of sea-ice around the Antarctic during that time.

The acarininid $\delta^{18} \mathrm{O}$ values during the Eocene were always considerably lower than those of the other planktonic species. Because the acarininids exhibit the lowest $\delta^{18} \mathrm{O}$ values of any of the taxa analyzed, we assume they were surface-dwelling forms. Notwithstanding the smaller amount of data available for the acarininids, it appears that the range of isotopic values exhibited by a single acarininid species throughout its stratigraphic range is less than that of Subbotina species. We interpret this to represent more restricted seasonal preferences and assume that the acarininids preferred the austral summer season when the thermocline was well developed. By contrasting the acarininid values, which are interpreted as summer surface-water temperatures, with the subbotinid and benthic isotopic temperatures, it is possible to derive an approximate measure of summer/winter seasonal temperature contrasts. During the late Paleocene this contrast appears to be have been between $2^{\circ}-3^{\circ} \mathrm{C}$. This seasonal contrast increased to between $4^{\circ}-5^{\circ} \mathrm{C}$ in the earliest Eocene and to $\sim 6^{\circ}-7^{\circ} \mathrm{C}$ at times during the late early Eocene.

\section{Middle Eocene: The Beginning of Climatic Cooling and Decreased Seasonality}

Beginning in the late early Eocene, at $\sim 53.0 \mathrm{Ma}$, the warm surface-water temperatures that had marked the late Paleocene and early Eocene began to decrease. This decrease continued through the middle and late Eocene with no apparent reversals. At this time there also appears to have been a reduction in the range of isotopic temperatures expressed by the subbotinid group. Because the range of subbotinid values is taken as a measure of overall seasonality, the reduction in isotopic variability in the middle Eocene is interpreted to reflect decreased seasonality in Antarctic surface waters. The decrease in seasonality corresponds to the beginning of the cooling trend that is recorded in all fossil groups and represents a long-term decrease in high latitude surface-water temperatures. Decreased seasonality can be expected to result from a progressive cooling of high latitude surface waters. By contrast, the acarininids show no clear evidence of decreased or increased variability and this suggests that these forms continued to be restricted to summer surface waters. The contrast between the maximum acarininid isotopic temperature values and the minimum subbotinid values provides a measure of summer/winter temperature contrast as described above. At $52.0 \mathrm{Ma}$ the maximum summer surface-water temperatures were $15^{\circ} \mathrm{C}$ and the minimum winter temperatures were $\sim 10^{\circ} \mathrm{C}$. By the middle middle Eocene $(\sim 45.0 \mathrm{Ma})$ maximum summer surface-water temperatures had decreased to $12^{\circ}-13^{\circ} \mathrm{C}$ and winter temperatures to $\sim 7^{\circ}-8^{\circ} \mathrm{C}$. These summer/winter temperature contrasts remained essentially the same during the early Eocene.

\section{Late Middle Eocene to Oligocene: Stepwise Cooling}

The cooling trend that began in the late early Eocene continued during the remainder of the Eocene. This cooling is largely encompassed within a series of steps at $43.0 \mathrm{Ma}, 40.0 \mathrm{Ma}$, and $\sim 36.0 \mathrm{Ma}$. Two of these are associated with sub-epoch boundaries at middle/late Eocene and the late Eocene/early Oligocene boundaries. The step at the Eocene/Oligocene boundary is well known. Less well known is the step at the middle/late Eocene boundary although it has been recognized previously by Keller (1983). These climatic steps can be recognized in the subbotinid group but the acarininids disappeared from Antarctic waters in the late middle Eocene, almost certainly related in some way to the climatic cooling. For this reason, it is not possible to contrast summer with winter sea-surface temperatures in the same way as is possible for the early Paleogene. Nonetheless, the magnitude of the climatic steps in the subbotinid record was $\sim 2^{\circ} \mathrm{C}$ at 43.0 and $40.0 \mathrm{Ma}$. At the Eocene/Oligocene boundary, the isotopic shift was twice that of the previous two steps. Since it is possible that ice accumulation began on the Antarctic continent at that time, the magnitude of the surface-water cooling cannot be easily estimated although it appears to have been no greater than $4^{\circ} \mathrm{C}$. The overall seasonality during the late middle and late Eocene, as measured by the variability expressed in the subbotinid isotopic record, was similar throughout this cooling interval and similar in magnitude to that at the beginning of the cooling trend.

Following the large isotopic shift at the Eocene/Oligocene boundary the subbotinid record exhibits a reversal in isotopic temperatures of $\sim 2^{\circ}-3^{\circ} \mathrm{C}$, peaking at $33.0 \mathrm{Ma}$ with a temperature of $\sim 5^{\circ} \mathrm{C}$. Because of the complication of possible continental ice accumulation these inferred temperatures near the Eocene boundary may be slightly in error. However, in general the minimum temperature recorded by the subbotinids was about $0^{\circ} \mathrm{C}$.

\section{Carbon Isotopic Changes}

The carbon isotopic record of the subbotinids exhibit values during the Paleogene that are similar, although slightly higher than benthic foraminifers (Kennett and Stott, this volume). The values are taken to represent averaged yearly $\delta^{13} \mathrm{C}$ conditions within the water column. As such they contain values influenced by summer conditions when the seasonal thermocline was well developed and winter conditions when the thermocline was broken down and the water column well mixed. By contrast the acarininids display $\delta^{13} \mathrm{C}$ values that represent surface conditions during the summer months (warmest $\delta^{18} \mathrm{O}$ values). We recognize four distinct stages in the history of the Antarctic seasonal thermocline based on the carbon isotopic patterns displayed by the subbotinids and acarininids (Fig. 6).

Stage One, during the late Paleocene through early Eocene: acarininids display significant $\delta^{13} \mathrm{C}$ separation and relatively high values compared with the subbotinids. This is inferred to represent a strong seasonal thermocline. This period appears to have been marked by particularly high summer surface-water productivity, resulting in more nutrient-depleted surface waters and consequently high $\delta^{13} \mathrm{C}$ values in the summer forms.

Stage Two, beginning in the latest early Eocene $(\sim 53.0 \mathrm{Ma})$ : the $\delta^{13} \mathrm{C}$ gradient between the acarininids and the subbotinids became less distinct. Acarininid $\delta^{13} \mathrm{C}$ values remained similar to those of the early Eocene, although there was slightly more variability. The subbotinids' $\delta^{13} \mathrm{C}$ values, relative to the acarininids, became more positive. This pattern suggests that the summer thermocline was still well established, but the possibility exists that the flux of organic carbon and biogenic carbonate was reduced, thereby reducing the vertical $\Delta \delta^{13} \mathrm{C}$.

Stage Three, beginning in the middle middle Eocene $(\sim 47.0$ $\mathrm{Ma})$ : the $\delta^{13} \mathrm{C}$ gradient between the subbotinids and the acarininids was further diminished. In addition, the acarininids display a greater range of values. In some instances the $\delta^{13} \mathrm{C}$ values of this group are similar to those of the subbotinids. These patterns indicate a further weakening of the summer thermocline and possibly greater variability in surface productivity.

Stage Four, during the late middle Eocene $(\sim 42.0 \mathrm{Ma})$; the acarininids disappeared from Antarctic waters. The evidence discussed above suggests that, in association with the long-term global cooling trend observed in the $\delta^{18} \mathrm{O}$ records, the thermocline progressively diminished. This may have occurred as a change in the duration and/or thickness of the summer thermocline (shorter summer thermal maxima). There may also have been greater intra-annual variation in the structure of the thermocline. The acarininids became extinct elsewhere soon after 


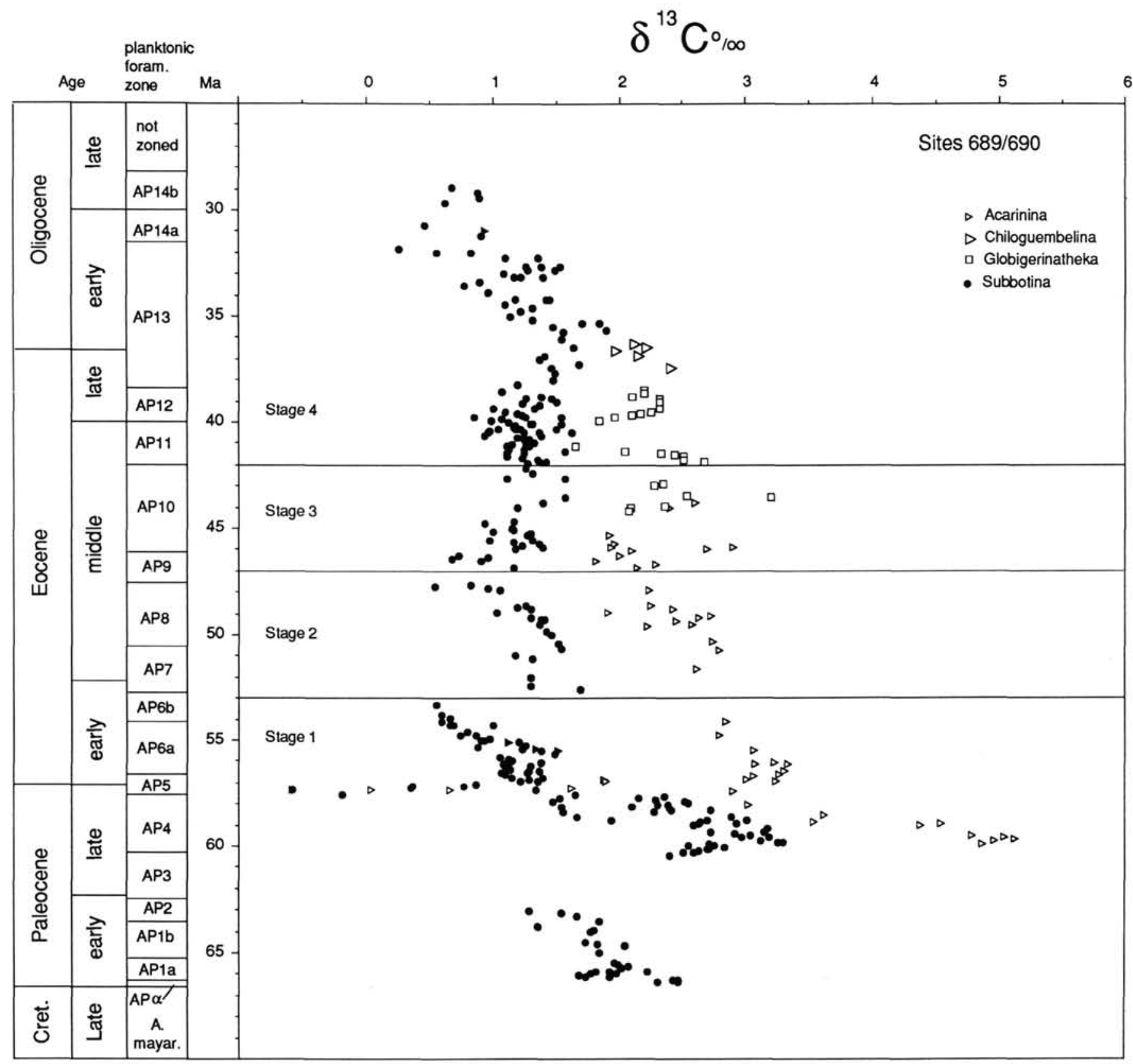

Figure 6. Composite carbon isotopic record of planktonic foraminifers (including Chiloquembelina cubensis and Globigerinatheka index) for ODP Sites 689 and 690. See Figure 1 for explanation. Four stages are shown representing inferred changes in the nature of the Antarctic thermocline (see text for discussion).

their disappearance in the Antarctic. The actual cause of the extinction is not known but may be related to factors associated with changes in the thermocline.

\section{CONCLUSIONS}

1. Oxygen and carbon isotopic composition of various Antarctic planktonic foraminifer taxa from Maud Rise has provided much new information on the evolution of sea-surface temperatures and the thermocline during the Paleogene.

2. Sea-surface temperatures have been estimated from planktonic $\delta^{18} \mathrm{O}$ values based on the assumption of no significant ice accumulation on the Antarctic continent before the earliest Oligocene, an assumption supported by independent Leg 113 evidence (Barker, Kennett, et al., 1988).
3. Acarininid carbon and oxygen isotopic values are inferred to reflect summer sea-surface conditions when the thermocline was well developed in the Antarctic Ocean. The subbotinids are inferred to have lived within the upper part of the water column throughout much of the year. As a group, their isotopic compositions record the range of seasonal variations within the upper part of the water column.

4. Maximum summer sea-surface temperatures in the Antarctic are recorded in the isotopic values of the acarininids. The subbotinids record minimum average temperatures within the upper part of the water column. The minimum subbotinid $\delta^{18} \mathrm{O}$ values are similar to those recorded by benthic foraminifers.

5. Average sea-surface temperatures were never less than $8^{\circ}-$ $9^{\circ} \mathrm{C}$ in Antarctica during the Paleocene and Eocene. Such warm 
temperatures would indicate a general absence of seasonal seaice in the vicinity of Maud Rise. During much of this time, seasurface temperatures were significantly warmer with estimated values up to $18^{\circ} \mathrm{C}$.

6. Sea-surface temperatures of the oceans surrounding Antarctica during much of the Paleocene-Eocene were temperate and at times approached cool-subtropical.

7. The overall climatic history of sea-surface conditions around the Antarctic can be summarized as follows: cool early Paleocene; warming during late Paleocene; climatic maximum in the early Eocene; followed by cooling during the remainder of the Paleogene, beginning in the early middle Eocene at $\sim 52.0 \mathrm{Ma}$. Superimposed on the Paleogene cooling trend were three discrete climatic steps, each associated with cooling of at least $3^{\circ} \mathrm{C}$. These occurred at $43.0,40.0$, and $\sim 36.0 \mathrm{Ma}$.

8. A dramatic warm spike involving the entire water column coincided with the Paleocene/Eocene boundary and major extinctions of deep sea benthic foraminifers. This event is attributed to a brief increase in the influence of deep waters formed in low latitudes.

9. The reduction of oxygen isotopic gradients between planktonic and benthic taxa at various times is inferred to represent a change in seasonal temperature contrasts. Seasonality was relatively low during the Paleocene, became higher during the early Eocene, and decreased through the remainder of the Paleogene.

10. The relationships between the oxygen and carbon isotopic records of acarininids and subbotinids show that the thermocline was well developed in Antarctica during the late Paleocene and early Eocene. The thermocline diminished in structure and/or duration through a series of stages associated with the long-term cooling during the remaining Eocene.

11. Changes in the nature of the thermocline in the Antarctic and possibly elsewhere may have influenced biotic evolution in the surface waters of the ocean during the Paleogene.

12. Stable isotopic composition of various planktonic foraminifer species have much potential in further defining the paleoceanographic evolution of the thermocline and climatic conditions at the surface of the Antarctic Ocean.

\section{ACKNOWLEDGMENTS}

We thank the scientific party of ODP Leg 113 for their collaboration. We thank R. Fairbanks, J. Zachos, and D. Pak for their assistance. J. Zachos and one other anonymous reviewer provided helpful suggestions which have improved the manuscript. The research was supported by USSAC grants FD 20103 and FD 20104 to L. Stott and J. Kennett and NSF grants (OCE 87-13391, OCE 88-17135, DPP 88-96183, and DPP-89-11554) to J. Kennett.

\section{REFERENCES}

Barker, P. F., Kennett, J. P., et al., 1988. Proc. ODP, Init. Repts., 113: College Station, TX (Ocean Drilling Program).

Bé, A.W.H., 1960. Ecology of recent planktonic foraminifera. Part 2. Bathymetric and seasonal distributions in the Sargasso Sea off Bermuda. Micropaleontology, 6:373-392.

1982. Biology of planktonic foraminifera. In Broadhead, T. W. (Ed.), Foraminifer: notes for a short course. Univ. of Tenn., Dept. of Geol. Sci., Stds. in Geol., 6:51-89.

Berggren, W. A., Kent, D. V., and Flynn, J. J., 1985. Paleogene geochronology and chronostratigraphy. In Snelling, N. J. (Ed.), The Chronology of the Geological Record, Mem. 10, Geol. Soc. London, 141-195.

Boersma, A., and Shackleton, N., 1979. Oxygen- and carbon-isotope variations and planktonic-foraminifer depth habitats, Late Cretaceous to Paleocene, Central Pacific, Deep Sea Drilling Project Sites 463 and 465 . In Thiede, J., Vallier, T. L., et al., Init. Repts. DSDP, 62: Washington (U.S. Govt. Printing Office), 513-526.

Douglas, R., and Savin, S., 1973. Oxygen and carbon isotope analyses of Cretaceous and Paleogene foraminifera from the central North
Pacific. In Winterer, E. L., Ewing, J. I., et al., Init. Repts. DSDP, 17: Washington (U.S. Govt. Printing Office), 591-604.

1975. Oxygen and carbon isotope analyses of Paleogene and Cretaceous microfossils from Shatsky Rise and other sites in the North Pacific Ocean. In Larson, R. L., and Moberly, R., Init. Repts. DSDP, 17: Washington (U. S. Govt. Printing Office), 509-520. 1978. Oxygen isotopic evidence for the depth stratification of Paleogene and Cretaceous planktic foraminifera. Mar. Micropaleontol., 3:175-196.

Keigwin, L. D., and Corliss, B. H., 1986. Stable isotopes in late middle Eocene to Oligocene foraminifera. Geol. Soc. Am. Bull., 97:335345.

Keller, G., 1983. Paleoclimatic analyses of middle Eocene through Oligocene planktic foraminiferal faunas. Palaeogeogr., Palaeoclimatol., Palaeoecol., 43:73-94.

Kennett, J. P., and Shackleton, N., 1976. Development of the psychrosphere $38 \mathrm{~m} . y$. ago: oxygen isotopic evidence from Subantarctic $\mathrm{Pa}$ leogene sediments. Nature, 260:513-515.

Miller, K., Janecek, T., Katz, M., and Keil, D., 1987. Abyssal circulation and benthic foraminiferal changes near the Paleocene/Eocene boundary. Paleoceanography, 2:741-761.

Mix, A. C., 1987. The oxygen-isotope record of glaciation. In Ruddiman, W. F., and Wright, H. E., Jr. (Eds.), North America and adjacent oceans during the last deglaciation, Geol. Soc. Am., 111-113.

O'Neil, J. R., Clayton, R. N., and Mayeda, T. K., 1969. Oxygen isotope fractionation in divalent metal carbonates. J. Chem. Phys., 51: $5547-5558$.

Reynolds, L., and Thunell, R. C., 1985. Seasonal succession of planktonic foraminifera in the subpolar North Pacific. J. Foraminiferal Res., 15:282-301.

Shackleton, N., 1974. Attainment of isotopic equilibrium between ocean water and the benthonic foraminifera genus Uvigerina: Isotopic changes in the ocean during the last glacial maximum. CNRS Res., Colloquium, 219:203-209.

Shackleton, N. J., Wiseman, J.D.H., and Buckley, H. A., 1973. Nonequilibrium isotopic fractionation between seawater and planktonic foraminiferal tests. Nature, 242:177-179.

Shackleton, N., and Kennett, J. P., 1975. Paleotemperature history of the Cenozoic and the initiation of Antarctic glaciation: oxygen and carbon isotope analyses in DSDP Sites 277, 279, and 281. In Kennett, J. P., Houtz, R. E., et al., Init. Repts. DSDP, 29: Washington (U.S. Govt. Printing Office), 743-755.

Shackleton, N., Hall, M., and Boersma, A. 1984. Oxygen and carbon isotope data from Leg 74 foraminifers. In Moore, T. C., Jr., Rabinowitz, P. D., et al., Init. Repts. DSDP, 74: Washington (U. S. Govt. Printing Office), 599-612.

Shackleton, N., Corfield, R. M., and Hall, M. A., 1985. Stable isotope data and the ontogeny of Paleocene planktonic foraminifera. J. FOraminiferal. Res., 15:321-336.

Smith, R. K., and Gowing, M. M., 1988. Mesopelagic and bathypelagic planktonic foraminifera from the North-central Pacific. Am. Zool., 28:32A.

Spero, H. J., and DeNiro, M. J., 1987. The influence of symbiont photosynthesis on the $\delta^{18} \mathrm{O}$ and $\delta^{13} \mathrm{C}$ values of planktonic foraminiferal shell calcite. Symbiosis, 4:213-228.

Spero, H. J., and Williams, D. F., 1988. Extracting environmental information from planktonic foraminiferal $\delta^{13} \mathrm{C}$ data. Nature, 335(6192):717-719.

Thunell, R. C., and Honjo, S., 1987. Seasonal and interannual changes in planktonic foraminifer production in the North Pacific. Nature, 328:335-337.

Williams, D. F., Sommer, M. A., II, and Bender, M. L., 1977. Carbon isotopic compositions of recent planktonic foraminifera of the Indian Ocean. Earth Planet. Sci. Lett. 36:391-403.

Woodruff, F., and Savin, S., 1989. Miocene Deepwater Oceanography. Paleoceanography, 4:87-140.

Zachos, J. C., Arthur, M. A., and Dean, W. E., 1989. Geochemical evidence for suppression of pelagic marine productivity at the Cretaceous/Paleogene boundary. Nature, 337:61-64.

Date of initial receipt: 7 April 1989

Date of acceptance: 10 October 1989

Ms 113B-187 